\title{
Black Holes and Neutron Stars in Vector Galileons
}

\author{
Javier Chagoya ${ }^{a}$, Gustavo $\mathrm{Niz}^{b}$, and Gianmassimo Tasinato ${ }^{a}$ \\ ${ }^{a}$ Department of Physics, Swansea University, Swansea, SA2 8PP, U.K. \\ ${ }^{b}$ Departamento de Física, Universidad de Guanajuato - DCI, C.P. 37150, León, Guanajuato, México.
}

\begin{abstract}
The direct detection of gravitational waves opens new perspectives for measuring properties of gravitationally bound compact objects. It is then important to investigate black holes and neutron stars in alternative theories of gravity, since they can have features that make them observationally distinguishable from their General Relativity (GR) counterparts. In this work, we examine a special case of vector Galileons, a vector-tensor theory of gravity with interesting cosmological properties, which consists of a one parameter modification of the Einstein-Maxwell action. Within this theory, we study configurations describing asymptotically flat, spherically symmetric black holes and neutron stars. The set of black hole solutions in this theory is surprisingly rich, generalising results found in GR or in related scalar-tensor theories. We investigate the properties and conserved charges of black holes, using both analytical and numerical techniques, highlighting configurations that are more compact than in GR. We then study properties of neutron stars, showing how the vector profile can influence the star internal structure. Depending on properties of matter and fields inside the star, neutron stars can be more massive than in GR, and they can be even more compact than Schwarzschild black holes, making these objects observationally interesting. We also comment on possible extensions of our configurations to magnetically charged or rotating configurations.
\end{abstract}

\section{Introduction}

The first direct detection of gravity waves opens a new window for astronomy [1], promising to measure with great accuracy properties of gravitationally bound compact objects. In this context, it is important to investigate general properties of black holes and neutron stars in alternative theories to General Relativity (GR). The aim is to understand whether the physics of compact objects allows one to distinguish among different theories of gravity, which may share similar predictions in the weak gravity regime but present a different behaviour when strong gravity is involved. The simplest and most popular modifications of GR are scalar-tensor theories, which have been extensively studied in stationary and cosmological situations (see [2] for a comprehensive review). Asymptotically flat black holes in scalar-tensor theories are usually similar to those in GR, thanks to powerful no-hair theorems that forbid the existence of scalar hair in black holes (see e.g. [3]). Instead, the physics of compact objects such as neutron stars can reveal interesting new effects, allowing one to distinguish them from their GR counterparts (see e.g. [4]).

In contrast, vector-tensor theories are relatively unexplored, although they can have important applications for cosmology. The standard Maxwell electromagnetism, vector field models of inflation [5], vectors mediating dark forces in millicharged dark matter scenarios [6], or vector dark energy set-ups [2] are examples of this fact. Recently, models of vector Galileons coupled with gravity have been introduced, where the vector Abelian symmetry is broken by derivative vector self-couplings, and its longitudinal polarization acquires Galilean interactions in the appropriate decoupling limit [7]. These set-ups are free of Ostrogradsky instabilities, and have been explored in a variety of contexts - see e.g. [8]. In this work, we focus on the physics of spherically symmetric black holes and neutron stars in one of the simplest vector-Galileon scenarios, described by the vector-tensor non-minimal coupling

$$
\beta \sqrt{-g} G_{\mu \nu} A^{\mu} A^{\nu},
$$

in addition to the standard Einstein-Maxwell Lagrangian. Here, $G_{\mu \nu}$ is the Einstein tensor, $A^{\mu}$ is the vector field, and $\beta$ is a constant parameter. This term is a special case of these vector Galileons, and the Abelian symmetry is only broken by the non-minimal coupling to gravity. As a consequence, the theory propagates five degrees of 
freedom: two tensors (gravity), two vectors (the vector transverse modes), and one scalar (the longitudinal vector polarization). The latter turns dynamical by the symmetry breaking term (1). We study asymptotically flat black holes and neutron stars, in the absence of a cosmological constant, pointing out the differences between these systems and their counterparts in scalar-tensor theories, where normally no-hair theorems prevent scalar hair to be detected asymptotically.

The set of black hole solutions in this theory is surprisingly rich, generalising not only the standard solutions of Einstein-Maxwell theory, but also the black hole configurations of related scalar-tensor theories. When studying neutron stars, we find that the vector plays an important role for determining the interior configuration of the star, and allows us to find examples of stable neutron stars that can be more massive, and more compact, than their GR counterparts. Whether the vector field is in the dark sector and plays a role in governing the dark energy or dark matter components, or if it corresponds to a modification of Maxwell's electromagnetism in the strong gravity regime, our findings may have relevant astrophysical applications.

The roadmap for this work is the following:

- Section 2 presents our system, some motivations, and the equations of motion to be examined.

- Section 3 studies spherically symmetric, asymptotically flat black hole configurations, going well beyond the results we first presented in [9]. When the parameter $\beta$ is turned on, we find two distinctive branches of asymptotically flat black hole solutions. The first branch is characterised by non-trivial profiles for the vector longitudinal and transverse polarizations, and the geometry is well described by a Schwarzschild configuration, plus subleading corrections which decay at large distances. Black holes are characterized by Komar charges corresponding to a mass and a vector charge, similarly to the Reissner-Nordström configurations in the Einstein-Maxwell theory. An additional integration constant, mainly controlling the profile of the longitudinal vector polarization, is not associated with an asymptotic conserved charge, hence it does not correspond to a black hole scalar hair. In the second branch of solutions, the configuration is more sensitive to the integration constants associated with vector and scalar profiles; they can modify the leading contributions to the geometrical black hole features, rendering the geometry very different from the Schwarzschild solution. We also study analytically how black hole configurations behave in the limit of $\beta$ going to zero, which requires some care due subtle strong coupling effects, which are nevertheless manageable in our context.

- Analytic solutions describing black holes are possible only for special choices of parameters, and more generally, a numerical analysis is needed. Therefore, in subsections 3.3 and 3.4 we numerically study some black holes and their properties, in cases where analytic solutions are absent. We analyse what conditions should be satisfied to obtain physically acceptable configurations. We find regions of the parameter space where regular black hole configurations exist, and more interestingly, where the compactness of such solutions can be much larger than in GR, making this vector Galileon model observationally distinguishable from GR in the black hole sector.

- Section 4 studies neutron stars, with particular attention to the role of the vector profile to specify the star configuration. The non-minimal coupling of equation (1) can influence the star's internal structure, since for certain values of the parameter $\beta$, the vector contributes to the energy momentum tensor and modifies the geometry. Moreover, neutron stars in this vector Galileon model can be larger and more massive than in GR, and, for certain parameter ranges, more compact. Actually, they could be even more compact than the Schwarzschild black hole for some cases, making these objects observationally interesting. We also comment on possible generalizations to magnetically charged or rotating configurations, which should be of interest in the case where the vector-tensor coupling (1) is considered a modification to Maxwell's electromagnetism, describing exotic objects such as magnetars.

- Section 5 is devoted to conclusions, with a summary of our main results, and a brief discussion of ways forward for testing vector-tensor theories in strong gravity regimes. Moreover, we include appendixes that contain technical details, or extensions to the material presented in the main text. 


\section{Set-up}

We consider a specific case of the theory of vector Galileons [7], which is characterized by the breaking of an Abelian symmetry due to a ghost-free non-minimal coupling of the vector field to gravity. The action is

$$
S=\int d^{4} x \sqrt{-g}\left[\frac{1}{2 \kappa} R-\frac{1}{4} F^{\mu \nu} F_{\mu \nu}+\beta G_{\mu \nu} A^{\mu} A^{\nu}+\mathcal{L}_{\text {matter }}\right] .
$$

$\kappa$ and $\beta$ are the gravitational and vector Galileon coupling constants, $F_{\mu \nu}=\partial_{\mu} A_{\nu}-\partial_{\nu} A_{\mu}$, and $G_{\mu \nu}$ is the Einstein tensor. The dimensions of the quantities involved are as follows: $R, G_{\mu \nu}$ have units of length ${ }^{-2}$, while $\beta$ and $g_{\mu \nu}$ are dimensionless. Using standard QFT conventions, $\kappa$ has units of length squared, and $c=1$. This system is invariant under inversion $A_{\mu} \rightarrow-A_{\mu}$; however, the Abelian symmetry, $A_{\mu} \rightarrow A_{\mu}+\partial_{\mu} \xi$ (with $\xi$ an arbitrary function) is broken by the non-minimal coupling between gravity and the vector, weighted by the parameter $\beta$.

Vector fields can play an important role for cosmology, even hypothetical ones that do not correspond to the carriers of Maxwell electromagnetic force. The vector-tensor set-up we consider in eq (2) belongs to a class of theories that have been studied in various works as a theory of dark energy. Scenarios involving dark vector fields associated with millicharged dark matter and dark photons are also sometimes analysed in the context of dark matter. Here we keep agnostic on the nature of the vector field, and we focus on how it backreacts to the gravity sector, determining the gravitational properties of compact objects. Switching on the non minimal coupling $\beta$ in the action (2) leads to a far richer variety of asymptotically flat black holes and neutron stars than configurations associated with the Einstein-Maxwell action.

The covariant field equations of motion (EOMs) corresponding to (2) are

$$
\begin{aligned}
0= & \frac{1}{2 \kappa} G_{\mu \nu}-\frac{1}{2}\left[F_{\mu \rho} F_{\nu}{ }^{\rho}-\frac{1}{4} g_{\mu \nu} F^{2}\right]+\beta\left[\frac{1}{2} g_{\mu \nu}\left(D_{\alpha} A^{\alpha}\right)^{2}-2 A_{(\mu} D_{\nu)} D^{\alpha} A_{\alpha}\right. \\
& +g_{\mu \nu} A_{\alpha} D^{\alpha} D^{\beta} A_{\beta}+\frac{1}{2} g_{\mu \nu} D_{\alpha} A_{\beta} D^{\beta} A^{\alpha}+D_{\alpha}\left(A_{(\nu} D_{\mu)} A^{\alpha}+A_{(\mu} D^{\alpha} A_{\nu)}-A^{\alpha} D_{(\mu} A_{\nu)}\right) \\
& \left.-2 D^{\alpha} A_{(\mu} D_{\nu)} A_{\alpha}-\frac{1}{2}\left(A^{2} G_{\mu \nu}+A_{\mu} A_{\nu} R-D_{\mu} D_{\nu} A^{2}+g_{\mu \nu} \square A^{2}\right)\right]-\frac{1}{2} T_{\mu \nu}, \\
0= & D^{\mu} F_{\mu \nu}+2 \beta G_{\mu \nu} A^{\mu},
\end{aligned}
$$

where

$$
T_{\mu \nu}=-\frac{2}{\sqrt{-g}} \frac{\delta\left(\sqrt{-g} \mathcal{L}_{\text {matter }}\right)}{\delta g^{\mu \nu}} .
$$

Notice that eqs (3a)-(3b) do not reduce to Maxwell equations when selecting the Minkowski metric and $T_{\mu \nu}^{(m)}=0$. Dynamical gravity sets constraints that the vector field has to satisfy, even around Minkowski's spacetime .

In what follows, we are interested in studying configurations that are static, spherically symmetric, and asymptotically flat. Asymptotic flatness is imposed in order to analyse in a more transparent way large distance properties (such as charges) of the solutions, and to appreciate the differences with scalar-tensor theories. In the first part of the paper, we focus our study to configurations in the absence of a matter energy-momentum tensor (that is, we set $\mathcal{L}_{\text {matter }}=0$ in eq $(2)$ ), while in the second part, we study the physics of compact objects corresponding to neutron stars (and we include an $\mathcal{L}_{\text {matter }}$ describing the stellar internal content).

Under our assumptions, we select the following general field $A n s a t z^{1}$ for determining spherically symmetric configurations

$$
\begin{aligned}
d s^{2} & =-\left(1-\frac{2 n(r)}{r}\right) d t^{2}+\left(1-\frac{2 m(r)}{r}\right)^{-1} d r^{2}+r^{2} d \theta^{2}+r^{2} \sin ^{2} \theta d \varphi^{2} \\
\mathbf{A} & =\left(A_{0}(r), \pi(r), 0,0\right)
\end{aligned}
$$

Notice that we allow for a non trivial profile $\pi(r)$ for the longitudinal polarization of the vector, which is not a pure gauge mode in this context, due to the broken Abelian symmetry.

\footnotetext{
${ }^{1}$ One may search for more general static configurations by choosing the possibility of time dependence in the vector field, while keeping the vector's effective energy-momentum tensor and the metric time-independent. However, one of the metric equations (3a) forces the longitudinal component $\pi$ to be a function of only the radial coordinate, while one of the vector's equations (3b) implies that $A_{0}$ cannot be a function of $t$ and $r$ simultaneously. If time-dependence in $A_{0}$ is chosen then a further constrain shows that it can only be a constant.
} 
Our study aims to theoretically examine the properties of regular black holes and neutron stars in scenarios where the Goldstone boson of a broken symmetry has Galileonic interactions, at least in an appropriate decoupling limit [7]. Our vector Galileon set-up shares this feature with the dRGT massive gravity, where black hole solutions have been found (see for example [10]). One advantage of vector Galileons to more complicated theories with vectors such as massive gravity is that black hole configurations are simpler to analyse, and one can analytically understand what happens to these vacuum configurations in the limit where $\beta$, the symmetry breaking parameter, goes to zero. As in other theories where there is no Birkhoff theorem, the black hole geometries found depend on various integration constants, and it is our aim to examine their physical interpretation.

The charged configurations that we will discuss may also have applications in astrophysics. In nature, astrophysical black holes and neutron stars are believed to be uncharged under Maxwell's electric field, because electrically charged black holes would absorb oppositely charged particles from their surrounding, loosing their own charge. In contrast, if these objects are charged under a dark vector field that is different from electromagnetism - motivated by dark sector theories as discussed above - then the standard model of particles does not feel its associated long range force, preventing a discharge of these configurations.

\section{Black Holes}

In this section, we determine and analyze solutions corresponding to charged black holes with a regular horizon. To make context with previous work on vector fields in gravity, the non-minimal vector-tensor coupling $\beta A^{\mu} A^{\nu} G_{\mu \nu}$ breaks the Abelian symmetry and acts as an effective mass for the vector on a given background. However, it differs from the usual mass term $m^{2} A^{2}$, thus avoiding Bekenstein's no-go theorems [11] against the existence of black hole solutions in Proca theories. See the discussion in [9] for further details. In our set-up, regular solutions do exist for general values of the coupling constant $\beta$ and, both the the vector profile $A_{0}$ (associated with an electric field $F_{0 i}$ ) and the scalar profile $\pi$ (the longitudinal vector polarization), play an important role in characterising these solutions.

When the parameter $\beta$ is set to zero, we recover the Einstein-Maxwell action, where there is a unique static, spherically symmetric, and asymptotically flat solution i.e. the Reissner-Nordström (RN) black hole configuration

$$
\begin{aligned}
d s^{2} & =-\left(1-2 M / r+\kappa Q^{2} / r^{2}\right) d t^{2}+\frac{d r^{2}}{\left(1-2 M / r+\kappa Q^{2} / r^{2}\right)}+r^{2} d \theta^{2}+r^{2} \sin ^{2} \theta d \varphi^{2} \\
A_{0} & =\frac{Q}{r}
\end{aligned}
$$

which corresponds to a charged black hole. In our case, when the single parameter $\beta$ is turned on, a much richer variety of black hole configurations exist with integration constants controlling vector and scalar configurations. We started the exploration of this topic in the work [9] by focusing on the specific case $\beta=1 / 4$. In this section we extend the analysis and show the existence and behaviour of solutions for general values of $\beta$ (see also [12, 13]).

There are two branches of solutions for our system. Substituting our Ansatz (5) in the equations of motion (3), we find, among other equations, the following key condition (obtained from the off-diagonal components of the Einstein equations):

$$
2 \beta \pi(r)\left\{n(r)-m(r)-[r-2 m(r)] n^{\prime}(r)\right\}=0 .
$$

When $\beta \neq 0$, this equation shows the existence of two distinct branches of solutions: one with a non trivial profile for the vector longitudinal polarization (controlled by the scalar $\pi(r)$ ); the other with $\pi(r)=0$. A similar situation - two different branches of spherically symmetric solutions - occur in many examples of modified gravity scenarios, from Gauss-Bonnet gravity [14] to massive gravity (see for example [10,15-17]). An interesting feature of our case is that both branches admit asymptotically flat configurations. This implies that there is no uniqueness theorem for spherically symmetric, asymptotically flat solutions in this theory. Each branch has distinctive features that we study separately ${ }^{2}$.

\footnotetext{
${ }^{2}$ The existence of two branches of solutions has been also pointed out in the recent paper [13], which we received while our work was finalised. (See also [12].) Our results are complementary to those presented in [13], where in addition, we numerically describe properties of black holes in the branch $\pi \neq 0$, analyse properties of vacuum configurations in the branch $\pi=0$, and study neutron star configurations.
} 
Given the special features and physical consequences found in the branch $\pi \neq 0$, we devoted the rest of the main sections to the study of this branch, and leave the discussion of the second branch, where $\pi=0$, to the appendix A. In Appendix $\mathrm{C}$ we instead discuss differences and similarities among vector-galileon and scalar-galileon black holes.

\subsection{Analytical solutions}

In the case $\pi \neq 0$, the constraint (8) imposes the following relation between the metric components

$$
m=\frac{n-r n^{\prime}}{1-2 n^{\prime}}
$$

A consequence of this algebraic contraint is that this branch is disconnected from the RN configuration (6), which does not satisfy (9). (RN configurations belong to the second branch of solutions, as we shall see.) Moreover, the polynomial curvature invariants (e.g. the Ricci and Kretschmann scalars) vanish at large $r$ for arbitrary power-law asymptotic profiles of the metric components when the relation (9) holds. This hints towards the existence of asymptotically flat solutions.

In addition, the radial component of Einstein equations is algebraic in $\pi$, with solution

$$
\pi^{2}=\frac{r}{4 \beta(r-2 n)}\left[2 A_{0}(1-4 \beta)\left(\frac{n A_{0}}{r-2 n}+\left(r A_{0}\right)^{\prime}\right)-\frac{r A_{0}^{2}\left[1+8 \beta\left(n^{\prime}-1\right)\right]}{r-2 n}-\left(r A_{0}\right)^{\prime 2}\right] .
$$

The requirement of having a positive right hand side in equation (10) will impose constraints on our configurations. Together with the Bianchi identity, these conditions reduce (3a)-(3b) to two independent equations for $A_{0}$ and $n$, which in the absence of matter reduce to

$$
\begin{aligned}
\xi_{\text {vac }}^{(1)} \equiv & \frac{4 \beta-1}{r}\left\{2 r^{2} \frac{d}{d r}\left[\frac{A_{0}\left(A_{0} r\right)^{\prime}}{r}\left(2 n^{\prime}-1\right)\right]+2 A_{0}^{2}\left(2 n^{\prime}-1\right)+A_{0}\left[3 r A_{0}-10 r\left(r A_{0}\right)^{\prime}\right] n^{\prime \prime}\right\} \\
& -\frac{4\left(2 n^{\prime}-1\right) n^{\prime \prime}}{\kappa}-3\left[\left(r A_{0}\right)^{\prime}\right]^{2} n^{\prime \prime}+2\left(2 n^{\prime}-1\right)\left(r A_{0}\right)^{\prime}\left(r A_{0}\right)^{\prime \prime}=0, \\
\xi_{\text {vac }}^{(2)} \equiv & A_{0}(4 \beta-1) n^{\prime \prime}+\left(r A_{0}\right)^{\prime} n^{\prime \prime}+\left(1-2 n^{\prime}\right)\left(r A_{0}\right)^{\prime \prime}=0 .
\end{aligned}
$$

These second order coupled equations admit asymptotically flat black hole solutions, characterized by three independent integration constants: a mass, a vector 'electric' charge ${ }^{3}$ that controls the profile of $A_{0}(r)$, and an independent scalar parameter which controls the profile of $\pi(r)$.

Exact solutions to eqs. (11a)-(11b) describing these black holes are not straightforward to obtain in general. Nevertheless, one can make progress analytically for some values of $\beta$, or in appropriate perturbative regimes. There are two special cases where one may find complete analytic solutions in the branch $\pi \neq 0$ : one for a specific choice of the coupling constant $\beta$ and the other for a particular value of the vector charge.

\section{Charged black hole solutions for $\beta=1 / 4$}

For the particular value of $\beta=1 / 4$ an exact solution to eqs. (11a)-(11b) was found in [9]:

$$
\begin{aligned}
n(r)=m(r) & =M, \\
A_{0}(r) & =\frac{Q}{r}+P, \\
\pi(r) & =\frac{\sqrt{Q^{2}+2 P Q r+2 M P^{2} r}}{r-2 M} .
\end{aligned}
$$

The integration constants $M$ and $Q$ represent the mass and electric charge of the configuration. Moreover, $P$ is a new integration constant, absent in the standard Reissner-Noördstrom configuration. It controls the asymptotic profile of $\pi$, which decays as $r^{-1 / 2}$ if $P \neq 0$, or as $r^{-1}$ if $P=0$. The dimensions of these constants are: $[M]=$ length, $[Q]=$ length $/[\sqrt{\kappa}]$ and $[P]=1 / \sqrt{\kappa}$.

\footnotetext{
${ }^{3}$ With electric charge we mean the charge associated with the $F_{0 i}$ components of the vector's field strength; we do not necessarily identify it with the charge of Maxwell's electric field.
} 
This configuration corresponds to the Schwarzschild metric, but with both profiles for $A_{0}$ and $\pi$ turned on. This is an example of a stealth solution, where the effective energy momentum tensor associated to the vector field vanishes and the metric reduces to the GR case. For further details on this solution we refer to [9]. ( See also $[12,13]$.)

It is interesting to study how the BH solution departs from the configuration (12a)-(12c), when $\beta$ is slightly different from $\beta=1 / 4$. To understand this regime, we build a series expansion in the parameter $(\beta-1 / 4)$, with the aim to examine how the location of horizons is affected. Details on the series expansion can be found in Appendix D: here we summarize our findings. The series expansion in $(\beta-1 / 4)$ is well defined below some critical radial value $r_{\text {crit }}$. As long as this critical radius is inside the horizon $r_{h}$, then we can trust the value of the position of horizons calculated perturbatively. The analysis simplifies in regimes where $P=0$ and $M / Q \ll 1$, where to obtain $r_{\text {crit }}<r_{h}$ one needs

$$
3|\beta-1 / 4| A_{0}\left(r_{h}\right)^{2} \kappa \ll 1 .
$$

In this case, the corresponding position of the single black hole horizon is given by

$$
r_{h} \approx 2 M+(\beta-1 / 4) \frac{\kappa Q^{2}}{M}+\mathcal{O}\left[(\beta-1 / 4)^{2}\right] .
$$

Therefore, as long as our choice of charges and parameters ensures that inequality (13) is satisfied at $r=r_{h}$, the position of the horizon gets indeed shifted when $\beta \neq 1 / 4$, by an amount depending on the vector charge. Although there is no analytical guarantee that higher order terms in the series do not spoil this result, we performed numerical checks confirming that these findings are correct.

As a consequence, we notice that vector Galileons can admit black hole solutions which are more compact than Schwarzschild, since they have a smaller horizon radius for the same black hole mass (more on this later).

\section{Solutions describing black holes with no vector charge (but with a scalar profile)}

A second analytic solution in this branch can be found when the integration constant $Q$ associated with the radial dependence of the vector time-like component vanishes: $Q=0$. The solution is given by

$$
\begin{array}{r}
n(r)=m(r)=M, \\
A_{0}(r)=P, \\
\pi(r)=\frac{1}{2} \sqrt{\frac{P^{2}(r-2 M)+8 M P^{2} r^{2}}{r(r-2 M)^{2}}} .
\end{array}
$$

This configuration is geometrically described by a Schwarzschild metric, and it is valid for any $\beta$. The solution depends on two quantities: the black hole mass, $M$, and the scalar parameter $P$. In this uncharged case, this is the same solution one finds for a scalar-tensor theory with non-minimal scalar-tensor coupling $\partial^{\mu} \phi \partial^{\nu} \phi G_{\mu \nu}$. We discuss the relation with scalar-tensor theories in full length in Appendix C.

\subsection{Approximate Solutions and conserved charges}

Besides the two analytic solutions we have described, we can obtain analytic results by considering perturbative expansions in appropriate parameters. We consider two examples with interesting physical consequences.

\section{Asymptotic expansion of solutions for large radial distances, and asymptotic charges}

We analyse the system of equations at large radial distances. In such regime, to determine the solutions for arbitrary $\beta$, we expand our fields as

$$
\begin{aligned}
1-\frac{2 n(r)}{r} & =1+\epsilon n_{1}(r)+\epsilon^{2} n_{2}(r)+\mathcal{O}\left(\epsilon^{3}\right), \\
A_{0}(r) & =P+\epsilon a_{1}(r)+\epsilon^{2} a_{2}(r)+\mathcal{O}\left(\epsilon^{3}\right),
\end{aligned}
$$


where $\epsilon$ is a dimensionless positive small parameter, controlling our asymptotic expansion in powers of $1 / r$. One should stress that the powers can be fractional. The small value of $\epsilon$ reflects the fact that we are far away from the source. We find the solutions

$$
\begin{aligned}
1-\frac{2 n(r)}{r} & =1-\epsilon \frac{2 M}{r}+\epsilon^{2} \frac{Q^{2} \kappa \delta}{2 r^{2}}+\mathcal{O}\left(\epsilon^{3}\right), \\
1-\frac{2 m(r)}{r} & =1-\epsilon \frac{2 M}{r}+\epsilon^{2} \frac{Q^{2} \kappa \delta}{r^{2}}+\mathcal{O}\left(\epsilon^{3}\right), \\
A_{0}(r) & =P+\epsilon \frac{Q}{r}+\epsilon^{2} \frac{P Q^{2} \kappa \delta \beta}{r^{2}}+\mathcal{O}\left(\epsilon^{3}\right),
\end{aligned}
$$

where the constant parameter $\delta$ is defined by

$$
\delta=(1-4 \beta)\left(1+8 \kappa \beta^{2} P^{2}-3 \kappa \beta P^{2}\right)^{-1} .
$$

Moreover, for the particular case of $P=0$, the scalar mode has the following form

$$
\pi(r)=\epsilon \frac{Q \sqrt{8-1 / \beta}}{2 r}+\epsilon^{2} \frac{M Q(12 \beta-1)}{2 r^{2} \sqrt{\beta(8 \beta-1)}}+\mathcal{O}\left(\epsilon^{3}\right)+\ldots
$$

while for $P \neq 0$, it reads

$$
\begin{aligned}
\pi(r)= & \sqrt{\epsilon}\left[\frac{2 P(M P+Q)}{r}\right]^{1 / 2} \\
& +\epsilon^{3 / 2} \frac{\left[32 M P(M P+Q)+Q^{2}\left(8-\beta^{-1}-4 P^{2}(1-4 \beta) \kappa \delta\right)\right]}{8 \sqrt{2 r^{3} P(M P+Q)}}+\mathcal{O}\left(\epsilon^{3}\right) .
\end{aligned}
$$

(Recall the dimensions of the quantities: $[M]=[\sqrt{\kappa} Q]=[r]$ ). As in the exact solution for $\beta=1 / 4$ of the previous section, $M$ and $Q$ are integration constants representing the mass and vector charge respectively, whereas the parameter $P$ controls the asymptotic profile of the longitudinal vector mode $\pi$.

This series expansion shows that there are two cases for which a Schwarzschild metric is exactly recovered in the weak-field limit: one is for $\beta=1 / 4$ and the other for $Q=0$, both discussed in Section 3.1 above. In all other cases, there are corrections which start at second in an expansion in inverse powers of the radius. This implies that these corrections are suppressed at large distances with small deviations from GR predictions. As a consequence, the computation of Komar charges related with the geometry and the conserved vector current $J^{\mu}=\nabla^{\nu} F_{\nu}{ }^{\mu}$ give the same results as for the standard Reissner-Nordström configuration (see e.g. [18]): the black holes are charaterised by the black hole mass $M$ and the vector 'electric' charge $Q$ (with the meaning explained in footnote 3 ) . The scalar parameter $P$, on the other hand, is not related with a scalar charge associated with any Gauss law that is valid asymptotically. Nevertheless, this parameter is important for determining properties of the solution, as the existence and position of horizons.

\section{Small $\beta$ expansion}

In theories where breaking a symmetry introduces additional degrees of freedom, it is interesting to ask whether there is a well-defined limit where the symmetry is recovered. The vDVZ discontinuity of the Fierz-Pauli massive gravity [19], or the strong coupling problem in Horava gravity [20] are good examples of how delicate such limit can be, since the theories become strongly coupled. In our scenario, the equations are sufficiently manageable that a full analytic study can be carried on. A naïve $\beta \rightarrow 0$ limit in the branch of solutions with $\pi \neq 0$ is not well-defined, because the scalar longitudinal component $\pi(r)$ of the vector field becomes imaginary. However, a consistent $\beta \rightarrow 0$ limit can be defined, if the vector charge $Q$ simultaneously goes to zero in an appropriate way. One should distinguish two cases: $\beta<0$ and $\beta>0$. In the case of $\beta<0$, the fields $A_{0}$ and $\pi$ admit the following solution for small $\beta$

$$
A_{0}=P+\sqrt{|\beta|} \frac{Q}{r}+\mathcal{O}\left(|\beta|^{3 / 2}\right)
$$




$$
\pi=\frac{1}{2} \sqrt{\frac{Q^{2}(r-2 M)+8 M P^{2} r^{2}}{r(r-2 M)^{2}}}+\mathcal{O}\left(|\beta|^{1 / 2}\right) .
$$

This configuration is valid for any $Q$ and $P$ as long as $r>2 M$. On the other hand, if $\beta>0$ we have

$$
\begin{aligned}
A_{0} & =P+\sqrt{\beta} \frac{Q}{r}+\mathcal{O}\left(|\beta|^{3 / 2}\right), \\
\pi & =\frac{1}{2} \sqrt{\frac{-Q^{2}(r-2 M)+8 M P^{2} r^{2}}{r(r-2 M)^{2}}}+\mathcal{O}\left(|\beta|^{1 / 2}\right),
\end{aligned}
$$

which requires $P \neq 0$ in order to keep $\pi$ real for any $r>2 M$. For small $\beta$, the metric reduces to a Schwarzschild configuration, plus small corrections

$$
\begin{aligned}
& 1-\frac{2 n(r)}{r}=1-\frac{2 M}{r}+\frac{\beta Q^{2} \kappa}{2 r^{2}}+\mathcal{O}\left(|\beta|^{3 / 2}\right) \\
& 1-\frac{2 m(r)}{r}=1-\frac{2 M}{r}+\frac{\beta Q^{2} \kappa}{2 r^{2}}-\frac{\beta M Q^{2} \kappa}{r^{3}}+\mathcal{O}\left(|\beta|^{3 / 2}\right),
\end{aligned}
$$

Hence, we learn that a vanishing $\beta$ limit is connected with a Schwarzschild configuration (and not with a RN solution), accompanied with a non-trivial profile for $\pi(r)$, that in any case is non-physical, being a gauge mode in such limit. In Appendix A we compare this small $\beta$ case with the same limit for the other branch of solutions with $\pi=0$.

\subsection{Numerical Solutions}

The analytical considerations we made in the previous subsections hint towards the existence of a variety of asymptotically flat charged black holes, whose properties depend on $\beta$, and on the integration constants involved. In this section, we numerically explore features of black hole solutions in cases where analytical solutions are not available. In Appendix B we analyze the asymptotic behaviour of solutions for large $r$, and even though the analysis is designed for the neutron star configurations that we will discuss in Section 4, the findings remain essentially valid also for the black holes discussed here. One of the main results of Appendix B is that asymptotically regular configurations exist only with a relatively small absolute value $|\beta|$. For excessively large values of this parameter, the right-hand-side of eq (10) becomes negative at large values of the radial coordinate; hence the scalar $\pi$ turns complex and the solutions become unphysical. For this reason, in this subsection we choose the representative value $\beta=-1 / 4$ to examine the existence and position of horizons and/or singularities depending on the values of the available integration constants. The value $\beta=-1 / 4$ is sufficiently different from the $\beta=1 / 4$ case we have studied at length with analytical methods, and at the same time, is sufficiently small (in absolute value) to avoid scalar singularities at large radial distances.

Hence we study eqs (11a)-(11b) numerically for $\beta=-1 / 4$. We first impose initial conditions on the profile of $g_{t t}, A_{0}$ and their derivative at some small value of the radius $r=r_{i}$. We choose this radius at scale $r_{i} \equiv 10^{3} \mathrm{Km}$, and integrate the equations outwards and inwards. We do not need to integrate $g_{r r}$, since it is determined by the algebraic constraint (9). The initial conditions for $g_{t t}$ and $A_{0}$ can be expressed in terms of three constants, $\mathcal{M}, \mathcal{P}$ and $\mathcal{Q}$, in the following way

$$
\begin{aligned}
& g_{t t}\left(r_{i}\right)=1-\frac{2 \mathcal{M}}{r_{i}} \\
& A_{0}\left(r_{i}\right)=\mathcal{P}+\frac{\mathcal{Q}}{r_{i}} \\
& A_{0}^{\prime}\left(r_{i}\right)=-\frac{\mathcal{Q}}{r_{i}^{2}} .
\end{aligned}
$$

Notice that the values of these integration constants do not necessarily coincide with the black hole Komar charges at large radial distances that were discussed in Section 3.2. Indeed, we have seen that the metric components and the time-like vector profile $A_{0}$ receive subleading corrections which scale as higher inverse powers of the radius (at most as $1 / r^{2}$ ). They are sufficient to modify the radial values of the charges. Nevertheless, we numerically checked that the mass $\mathcal{M}$ of the configuration is barely affected by the radial evolution, thus we fix its initial 
value to $\mathcal{M}=4.5$ solar masses (from now on we denote solar masses with the symbol $M_{\odot}$ ), that approximatively corresponds to the asymptotic value of this quantity. To analyze the black hole properties, we vary the quantities $\mathcal{Q}, \mathcal{P}$, and explore under which conditions we obtain configurations corresponding to regular black holes. We look for geometries that are regular everywhere, besides from an essential singularity at the origin, which is covered by an event horizon located at the radial coordinate $r_{h}$ where

$$
g^{r r}\left(r_{h}\right)=0 .
$$

Moreover, regular configurations require that the scalar field is well defined outside the horizon, without turning complex (as discussed above). Our results are summarised in Figure 1, where we identify four qualitatively distinct regions of parameters.
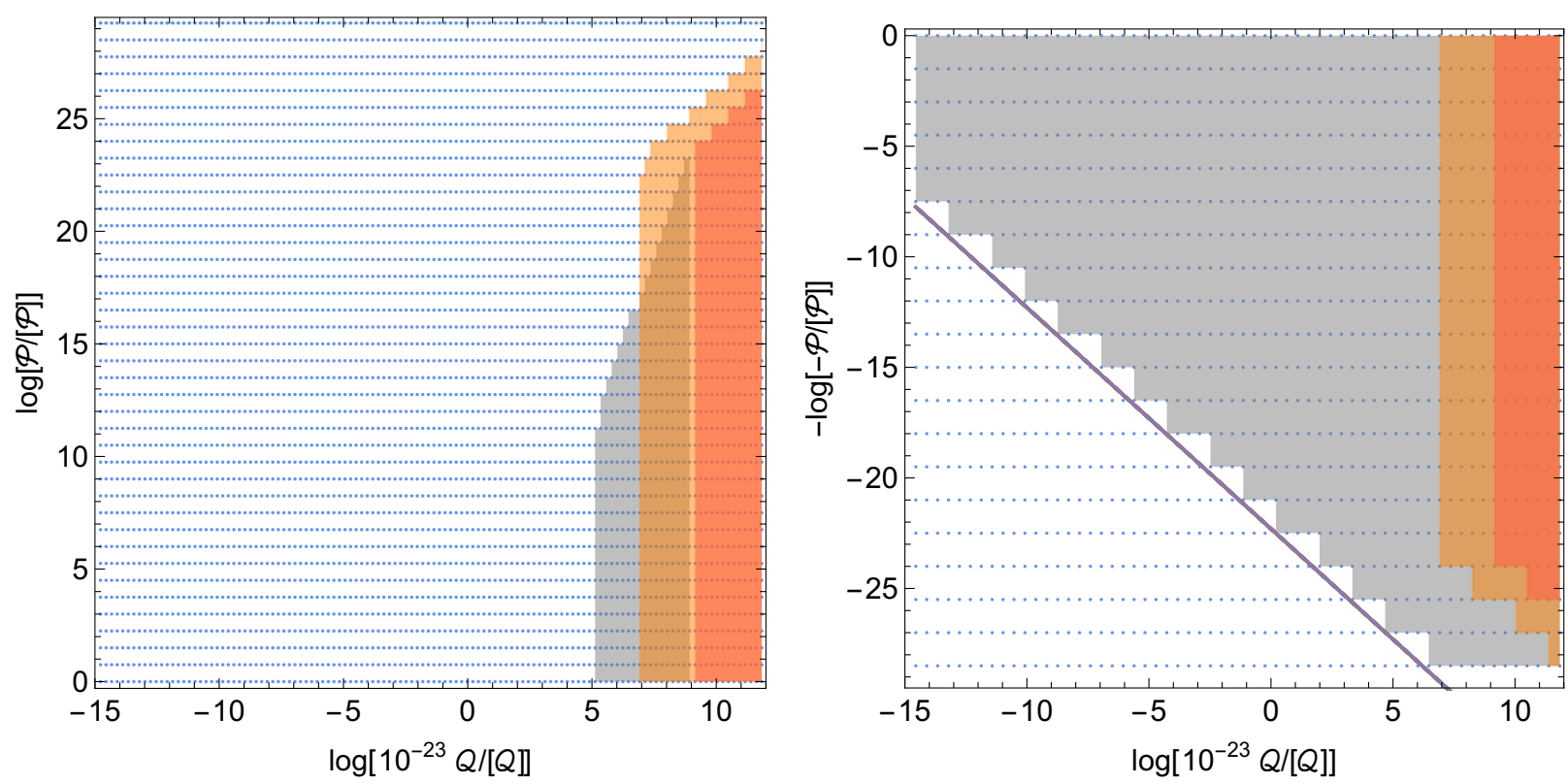

Figure 1: This figure represents the black hole behaviour in terms of the initial conditions $\mathcal{P}$ and $\mathcal{Q}$ imposed at $r_{i}=$ $10^{3} \mathrm{Km}$. In the horizontal and vertical axis we use logarithmic scales: the normalisation factor $10^{-23}$ is introduced for convenience, and the $[\mathcal{P}]$ and $[\mathcal{Q}]$ factors are used to make the argument of the logarithm dimensionless. Each point in the diagram represents one solution: by varying $\mathcal{P}$ and $\mathcal{Q}$, we identify four qualitatively different examples of configurations. The color notation indicates how the solution behaves: orange (R1) $\rightarrow$ no event horizon, red $(\mathrm{R} 2) \rightarrow$ singularity at large $r$, grey $(\mathrm{R} 3) \rightarrow$ complex $\pi$, and blue $(\mathrm{R} 4) \rightarrow$ regular solutions. All these solutions have $\mathcal{M}=4.5 M_{\odot}$. In the left panel we show the case when $\mathcal{P}$ and $\mathcal{Q}$ have the same sign, and in the right panel the case when they have opposite signs. The brown line in the right panel is the analytic limit $M P+Q=0$ obtained from eq (20a) for solutions with real $\pi$. See the main text for more details.

R1 (orange): Asymptotically flat metrics with a naked singularity at the origin: The shaded orange region corresponds to solutions that do not have an event horizon. Their inwards evolution shows that $g^{r r}$ does not vanish at any $r$. Instead the configuration diverges as $r \rightarrow 0$, where the Ricci scalar also diverges, indicating that it is an essential singularity. For large $r$, the solutions are asymptotically flat.

R2 (red): Naked singularities at the origin and at a finite radius: In the red shaded region we indicate solutions that do not have an event horizon, and are not well behaved asymptotically, since the Ricci scalar also diverges at finite large values of $r$.

R3 (grey): Black hole solutions with complex $\pi$ : The region indicated in grey includes solutions where $\pi$ becomes complex. We determine them by sampling the value of $\pi$ at different distances from the source, for all the solutions with an event horizon.

R4 (blue): Black hole metrics with real $\pi$ everywhere: This region is represented with blue points. It includes all the regular BH solutions with an event horizon, and with an everywhere well defined scalar $\pi$. 
This numerical analysis shows that the condition of having a regular configuration - equipped with a horizon and an everywhere well defined value of the scalar field - singles out a well defined region in the space of parameters $\mathcal{P}, \mathcal{Q}$. The boundaries among these regions in parameter space might contain interesting physics, as we will see in the next subsection.

While in this subsection we studied the dependence of the black hole properties from the integration constants $\mathcal{P}, \mathcal{Q}$ defined at small radial distances, we also repeated the analysis leading to Figure 1 considering boundary conditions evaluated far from the origin, where the corresponding integration constants are more directly related with black hole charges. We present the details of this discussion in Appendix E.

\subsection{Black hole compactness}

The black hole compactness, that we refer to by the letter $C$, is an important property to characterise black holes. It is defined in terms of the ratio of the black hole mass versus the position of the horizon (in Planck units). For the most famous (and relevant for our discussion) black hole solutions its corresponding values are

$$
\begin{aligned}
C & =\frac{1}{2} \quad \text { Schwarzschild BH } \\
\frac{1}{2} \leq C \leq 1 & \text { RN BH, upper bound saturated for extremal BH } .
\end{aligned}
$$

Given that detection of gravitational waves from black hole mergers can allow one to reconstruct geometrical properties of black holes as their size and mass, it is important to examine whether our set-up leads to values of $C$ that can be different from the above ones.

The answer is affirmative, and we examine two aspects of the question. First, we focus on the $\beta=-1 / 4$ case of Section 3.3. If we return our attention to Figure 1, at the boundaries between the regular region equipped with horizons, denoted in blue, and the region where horizon are absent, in orange, we might expect to find values of $r_{h}$ that are small, suggesting a potentially large $C$. This is confirmed in Figure 2 where it is shown that by choosing appropriately the values of the available integration constants, we can get configurations with large compactness $C$ - up to twice the value of $\mathrm{RN}$ black holes - in the proximity of the boundaries between regions.

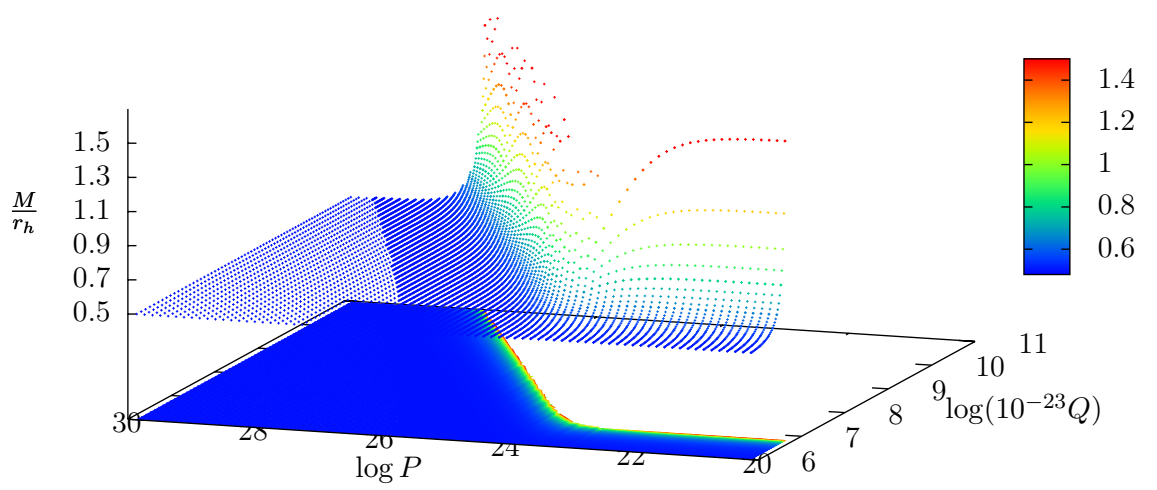

Figure 2: Vector Galileon black holes with high compactness. For all the solutions in this plot the black hole mass is $M \approx 4.5 M_{\odot}$. The colour map at the bottom is intended to help to visualise the borders of this region and the zones of high compactness, the empty part corresponds to R1 in the notation of Section 3.3, where the solutions do not have an event horizon.

A second possibility for studying black hole compactness consists in fixing the asymptotic integration constants to representative values and vary the coupling constant $\beta$, while numerically searching for the values of maximum compactness. We present the results of this search in Figure 3, which shows that maximal compactness depends in a non-trivial way on the value of $\beta$. For a choice $\log \left(10^{-23} Q /[Q]\right)=10.6, \log (P /[P])=27.5$, and $M=4.5 M_{\odot}$, the compactness is of order $C \simeq 2$ in proximity of $\beta=-1 / 4$.

Our numerical analysis shows that that there are regions in the available parameter space where vector Galileons can lead to black holes that are more compact than standard GR configurations. It would be interesting to further analyse the properties of these highly compact black holes, such as their stability and possible observational consequences in dynamical situations, such as binary mergers. 

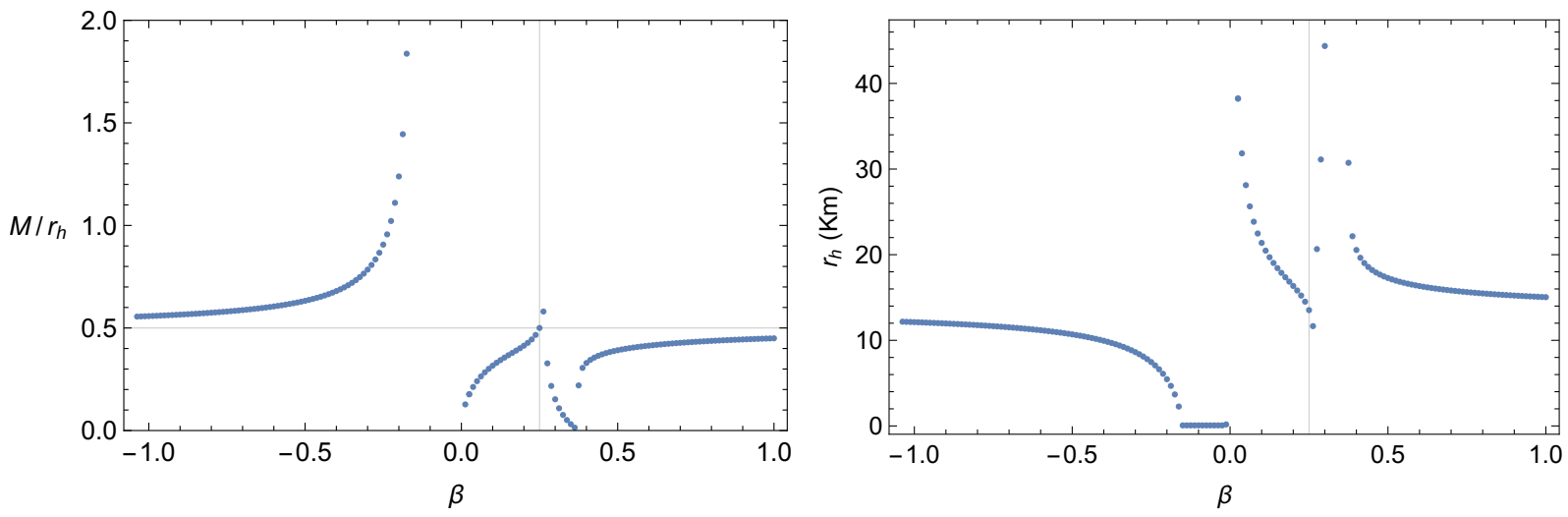

Figure 3: Compactness for different $\beta$ 's at fixed $P=3.1 \times 10^{17} \mathrm{~cm}^{1 / 2} \mathrm{gr}^{1 / 2} / \mathrm{s}, Q=4.1 \times 10^{33} \mathrm{~cm}^{3 / 2} \mathrm{gr}^{1 / 2} / \mathrm{s}$ and $M=4.5 M_{\odot}$. The points at $r_{h}=0$ do not correspond to black holes but to naked singularities at the origin.

\section{Neutron stars}

Neutron stars (NS) represent a promising arena for studying gravity in a strong field regime, and for revealing departures from GR in modified gravity scenarios (see e.g. [4,21] for reviews). For example, in the context of scalar-tensor Brans-Dicke theories, Damour and Esposito-Farese have pointed out the phenomenon of spontaneous scalarization of NS [22]. This effect can lead to large deviations from GR predictions, even in theories that satisfy PPN constraints in a weak field regime. The physics of neutron stars have also been studied within the Horndenski scalar-tensor framework. In particular, for a non-minimal derivative coupling of the scalar field to the Einstein tensor, by tuning the scalar field coupling constants and charge it has been numerically shown that it is possible to obtain NS's with masses up to approximately $2.5 M_{\odot}[23,24]$. In this case, the fields outside the NS's are described by the stealth Schwarzschild solution. NS's are also known in Einstein-Dilaton-Gauss-Bonnet gravity (see [25] and references therein), which is another sub-sector of Horndeski where the scalar field couples linearly to the Gauss-Bonnet term. See $[23,26]$ for reviews.

In this section we study NS solutions in our vector Galileon scenario, with the main aim to find NS systems with distinctive properties that make them distinguishable from GR. We show that the additional vector field profile associated with $A_{0}$ can lead to consistent NS configurations that are more massive and more compact than their GR counterparts. We focus on static, electrically charged configurations, and towards the end of this section we also comment on the possibility to include rotation and magnetic fields. This is interesting in the case where the non-minimal vector-tensor coupling is considered as modification of Maxwell electromagnetism in strong gravity regime.

In order to analyse NS configurations, we need to first determine solutions for the field profiles in the exterior of the star. These configurations are then smoothly connected with interior solutions, which depend on the equation of state of the internal matter. Interior solutions are determined by hydrostatic equilibrium configurations controlled by the matter which forms the star, and by the theory of gravity under consideration - in our case a vector Galileon model. For definiteness, we focus on the $\pi \neq 0$ branch of solutions of the constraint condition (8). In this branch, the geometrical exterior solutions are well described by the Schwarzschild geometry, plus corrections that rapidly decay at large distances, even in presence of non-trivial vector and scalar profiles, as we have discussed in the previous sections. Moreover, the analysis of Appendix B shows that, for this branch of solutions, the value of $\beta$ can not be too large in absolute value, otherwise the scalar field profile $\pi$ becomes complex in the exterior of the star. To avoid this, we make ${ }^{4}$ the same choice $\beta=-1 / 4$ we adopted for the numerical analysis of black hole solutions in Section 3.3. For this value of $\beta$ we find physically interesting NS solutions with sizeable differences from their GR counterparts. We checked that if $\beta$ is taken positive one does not find configurations that significantly different from GR.

Before proceeding, it is worth to remark that in the main text we do not consider direct couplings between the vector Galileon field and regular matter. In other words, we assume that the only interaction between the star's

\footnotetext{
${ }^{4}$ Notice that the analysis of Appendix B uses values of neutron star properties - radius, mass - corresponding to neutron star objects similar to GR. As we will learn in this Section, for objects with more exotic mass or radius one can find unphysical cases where the scalar turns complex even for $\beta=-1 / 4$.
} 
energy density and the vector field is gravitational. At the end of this section we discuss options for going beyond this approximation.

For describing matter inside a neutron star, we follow Damour and Esposito-Farese work [27] and use a polytropic Ansatz. We parametrise the equation of state in terms of a dimensionless function $\chi(r)$ as

$$
\begin{aligned}
& \rho(r)=\rho_{0}\left(\chi+\frac{K}{\Gamma-1} \chi^{\Gamma}\right), \\
& p(r)=K \rho_{0} \chi^{\Gamma}
\end{aligned}
$$

with $\rho_{0}=c^{2} n_{0} m_{b}=c^{2} 1.66 \times 10^{14} \mathrm{gr} / \mathrm{cm}^{3}$. $n_{0}$ is the baryon number density and $m_{b}$ the baryon mass. The polytropic constant $K$ and exponent $\Gamma$ take values appropriate to adjust the observed masses of NS. In GR, the values ${ }^{5} \Gamma=2.34$ and $K=0.0225$ give a mass-radius (M-R) curve with a maximum gravitational mass of 2.05 solar masses, in agreement with the largest observed neutron star masses (PSR J1614-2230 with $M=1.97 \pm 0.04$ Solar masses [28] and PSR J0348+0432 with $M=2.01 \pm 0.04$ Solar masses [29]). We have verified that the qualitative features of our configurations do no change if a more accurate equation of state is considered.

The covariant equations of motion (3) in the case of the neutron star configurations we are considering simplify in the following way. In the interior of the star, there are two algebraic conditions which determine the profiles for the metric component $m(r)$ and the scalar field $\pi(r)$, given by

$$
\begin{aligned}
m(r)= & \frac{n-r n^{\prime}}{1-2 n^{\prime}}, \\
\pi^{2}(r)= & \frac{r}{4 \beta(r-2 n)}\left[2 r^{2}\left(1-2 n^{\prime}\right) p-2 A_{0}(4 \beta-1)\left(\frac{n A_{0}}{r-2 n}+\left(r A_{0}\right)^{\prime}\right)\right. \\
& \left.-\frac{r A_{0}^{2}\left[1+8 \beta\left(n^{\prime}-1\right)\right]}{r-2 n}-\left(r A_{0}\right)^{\prime 2}\right] .
\end{aligned}
$$

Moreover, we have dynamical equations for the remaining quantities, which have the same structure as in the exterior of the object, but with contributions associated with the star energy momentum tensor, namely

$$
\begin{aligned}
& 0=\xi_{\text {vac }}^{(1)}-\frac{2 r^{2} \rho\left(2 n^{\prime}-1\right)^{3}}{r-2 n}+2 p r\left(2 n^{\prime}-1\right)\left[\frac{\left(2 n^{\prime}-1\right)\left(4 n-3 r+2 r n^{\prime}\right)}{2 n-r}-r n^{\prime \prime}\right], \\
& 0=\xi_{\text {vac }}^{(2)}
\end{aligned}
$$

where $\xi_{v a c}^{(1)}$ and $\xi_{v a c}^{(2)}$ are defined in eq (11a) and (11b). Together with the equation of state (30a-30b), and the energy-momentum tensor conservation, $\nabla_{\mu} T_{\nu}^{\mu}=0$, these equations further determine the time-like component $A_{0}$ of the vector, the 00-component of the metric (the function $n(r)$ ), and the density and pressure of matter.

We numerically solve these equations imposing appropriate boundary conditions, and choosing the value $\beta=$ $-1 / 4$ for the vector Galileon coupling constant. The condition that the metric is well behaved at the center of the star, $n(r=0)=0$, requires $A_{0}^{\prime}(0)=0$. We are left with only one free parameter to fully determine the initial condition at the origin: $A_{0}(r=0) \equiv A_{0 c}$. To work with dimensionless quantities, it is convenient to parametrise the initial conditions for $A_{0 c}$ as

$$
a_{0 c}=A_{0 c} /\left(10^{23} \mathrm{~cm}^{1 / 2} \mathrm{gr}^{1 / 2} / \mathrm{s}\right),
$$

so that $a_{0 c}$ is dimensionless and its logarithm of order 1 . Our aim is to determine how NS configurations depend on $a_{0 c}$, and how the results differ from standard GR configurations. We match the interior solution at a star radius $R_{\star}$ with a regular, asymptotically flat exterior solution for the field equations. The radius $R_{\star}$ corresponds to the value of the radial coordinate where the internal pressure vanishes. Figure 4 shows our results in terms of mass-radius (M-R) curves for different initial conditions $a_{0 c}$. Vector Galileons allow neutron star masses and radii larger than in GR, whose permitted configurations are represented with a black dashed line in Figure 4. The field profiles are well defined for any $a_{0 c} \geq 5$, and our configurations are stable according to the static stability criterion $d M / d \rho_{c}>0$ [30]. For $a_{0} \gtrsim 10$ we get masses larger than the upper bounds theoretically estimated in GR for any EoS [31]. Given the additional degree of freedom, it is expected that modified gravity models may help to relax the GR mass bounds [32].

\footnotetext{
${ }^{5}$ We re-adjust the value $K=0.0195$ used in [27] in order to account for updated observations on the maximum mass of neutron stars.
} 


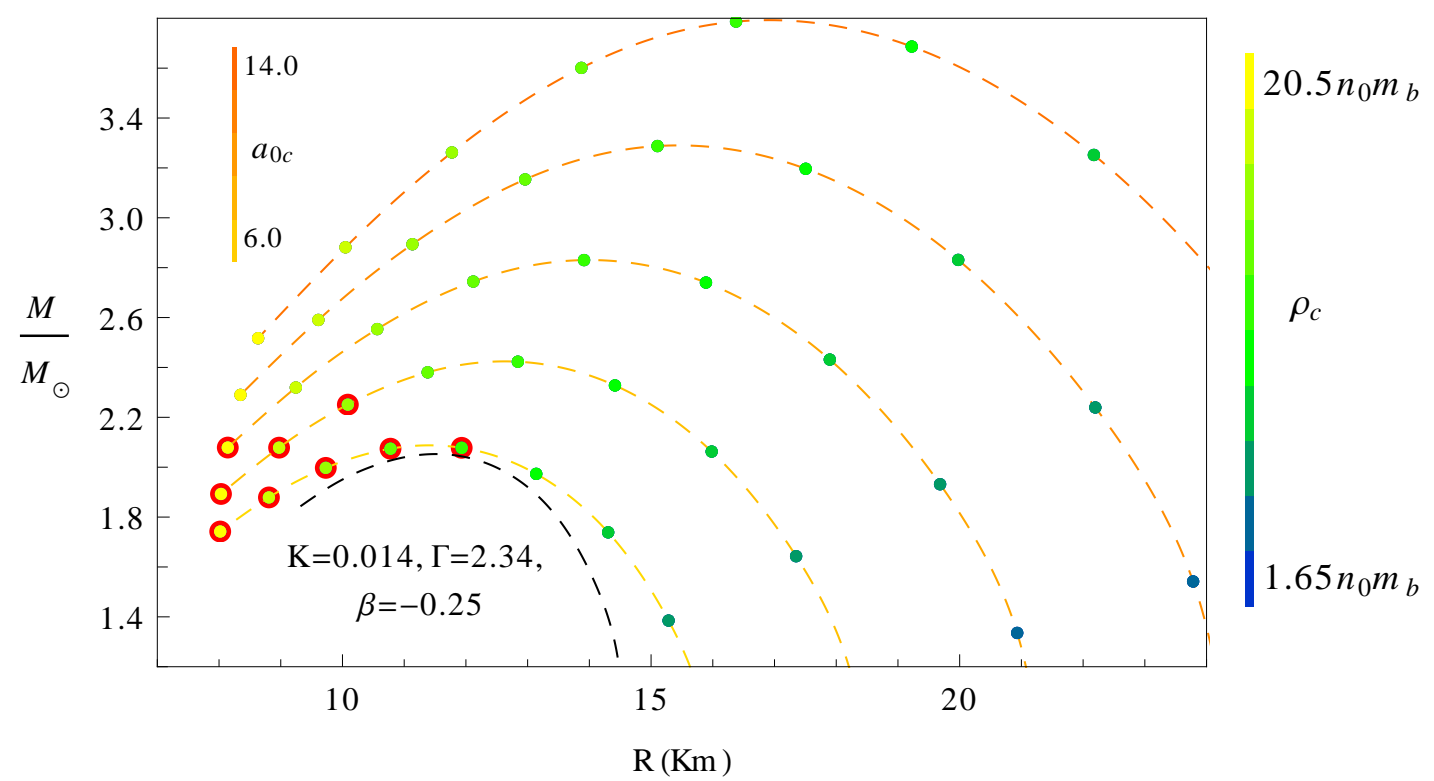

Figure 4: Mass-radius curves for neutron stars in vector Galileons for $\beta=-1 / 4$. We use a polytropic EoS with $K=0.014$ and $\Gamma=2.34$. Each line corresponds to solutions with the same central value $a_{0 c}$ indicated by the colour bar on the top left corner of the plot, and each point along these lines corresponds to a solution with central density $\rho_{c}$ indicated by the sidebar. The dark dashed line is the $M-R$ curve adjusted in GR to fit the maximum observed mass of a NS, it is computed using a polytropic EoS with $K=0.0225$ and $\Gamma=2.34$. This plot shows that for $\beta=-1 / 4$ we can obtain equilibrium configurations with values of $m\left(R_{*}\right)$ larger than in GR. Circled points correspond to unphysical configurations where the scalar field turns complex inside the star radius.

\subsection{Neutron star configurations when varying $a_{0 c}$}

The initial condition corresponding to the value of the vector profile at the origin, $a_{0 c}$, represents the key parameter for controlling the NS solutions. As we decrease $a_{0 c}$, maintaining the same values for the EoS parameters, we find that the scalar $\pi$ turns complex, hence the configuration has to be discarded.

At first sight, Fig. 4 would seem to suggest that NS solutions exist for arbitrarily large values of $a_{0 c}$. However, this is not always the case. In Fig. 5 we explore a larger range of values of $a_{0 c}$ for fixed central NS densities approximately up to the critical density associated with maximal mass in the $M-R$ curves. We find that the properties of solutions with high $a_{0 c}$ depend on $\rho_{c}$, the value of the density at the center of the star. For low densities, there are equilibrium configurations in the complete range of $a_{0 c}$ that we explored. Instead, for relatively high densities we find an upper bound on $a_{0 c}$ beyond which the radius of the star cannot be defined in the standard way, since we do not find any radial position $R_{\star}$ where the pressure of matter vanishes, hence the star has no natural boundary.

There exist additional constraints on the allowed range for $a_{0 c}$, which arise when studying in detail the external field configurations. When a low central density $\rho_{c}$ is chosen all the solutions can be matched to healthy exterior solutions. But when $\rho_{c}$ is increased, this is not the case. The black points in the left panel of Fig. 5 correspond to interior solutions that can not be matched to an asymptotically flat exterior spacetime, the black lines in the right panel show that the scalar field profile $\pi$ diverges for these solutions.

Interestingly, by calculating the NS mass using asymptotic properties of the exterior solutions, we always find a NS mass larger than in GR. We exemplify this fact in Fig. 6.

\subsection{Neutron star compactness}

As well as in the black hole case, the compactness of neutron stars is an important property that can be used for observationally characterizing these objects. In this subsection we show that, despite the constraints one has to satisfy, the exterior solutions of NS lie in a region of the parameter space where the NS compactness can be increased with respect to GR. 

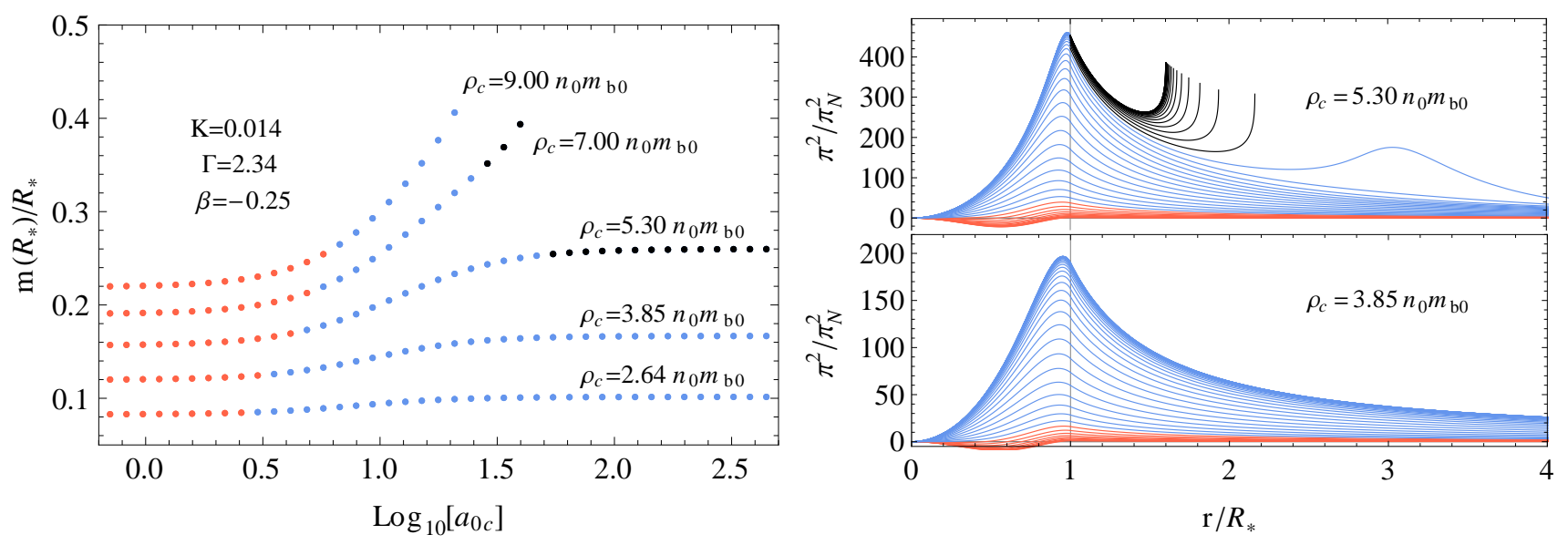

Figure 5: Range of interest for $a_{0 c}$. Each point in the left panel corresponds to a static configuration computed with the central density shown in the plot. At high densities there is a maximum $a_{0}$ beyond which star configurations do not exist. Red points represent solutions for which $\pi$ is complex, blue points regular solutions, and black points solutions that cannot be matched to a regular exterior solution. The right panel shows the interior and exterior scalar field profiles for configurations with the same central density, each profile is associated to a point in the left panel. For $a_{0 c} \lesssim 1, m\left(R_{*}\right)$ and $R_{*}$ remain constant. For $a_{0 c} \gtrsim 10^{2}$ and low densities, $m_{*}$ and $R_{*}$ increase with $a_{0 c}$, but the ratio $m_{*} / R_{*}$ remains constant.

In GR the exterior solution of a NS is exactly Schwarzshild. The compactness is then determined by dividing the Schwarzschild mass (in units of distance) over the radius of the star, and since $R$ is always larger than the Schwarzschild radius the compactness is always less than that of a Schwarzschild black hole, i.e., $1 / 2=M / r_{s}>$ $M / R$.

In vector Galileons, as shown in the previous sections, the asymptotic mass gets contributions from the vector field outside the star which can assume a non-trivial profile. Therefore, in order to obtain the compactness of a NS we first extract $M$ from the exterior solution evaluated at a large $r$ - where the asymptotic Schwarzschild solution holds - and then divide it by the radius of the star. The result is always smaller than the compactness of a vector Galileon black hole. This is expected, but interestingly, the compactness of such black holes can be larger than that of a Schwarzschild BH, opening the possibility for NS in vector Galileon theories that are more compact than GR black holes.

In Fig. 7 we represent the results of a numerical analysis aimed to exemplify the discussion above. Each point in the plot corresponds to a global solution (i.e., a NS plus the exterior solution) with initial conditions indicated by
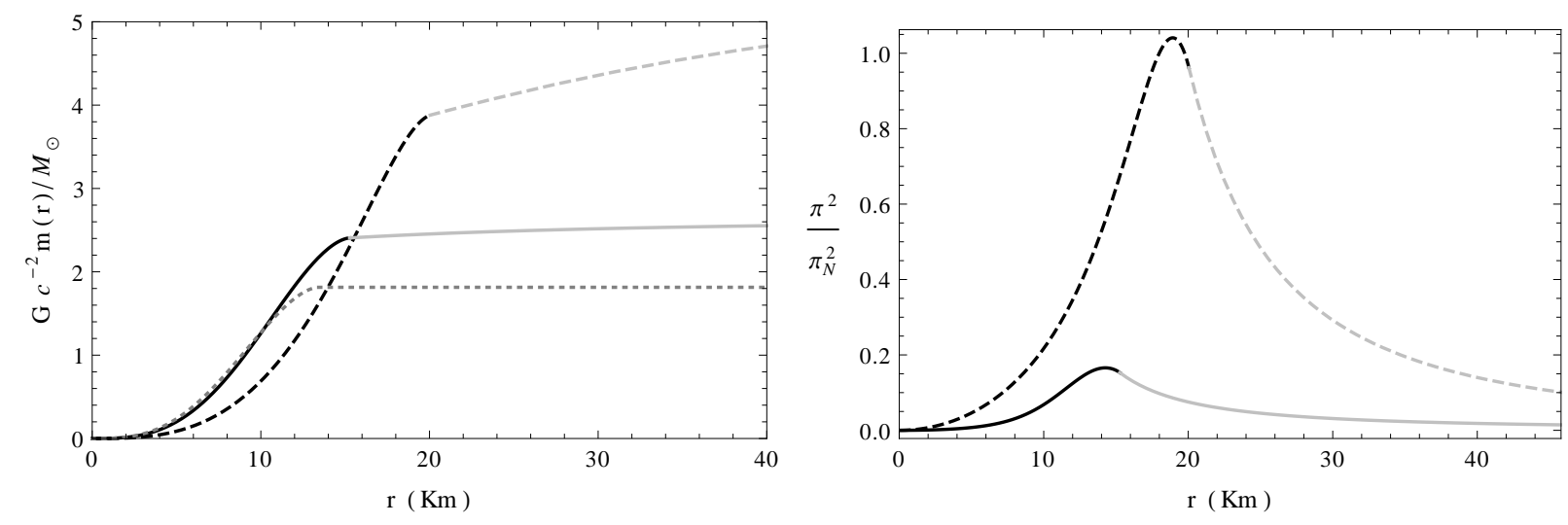

Figure 6: Interior (black line) and exterior solutions (grey line) for $\beta=-1 / 4$. Solid lines are for $a_{0}(0)=6.0$ and dashed lines for $a_{0}(0)=12.0$. The central density is $\rho_{c}=5.3 n_{0} m_{b 0}$. The left panel shows $m(r)$ in units of solar masses, both lines reach asymptotic constant values. The dotted line is the GR solution. 


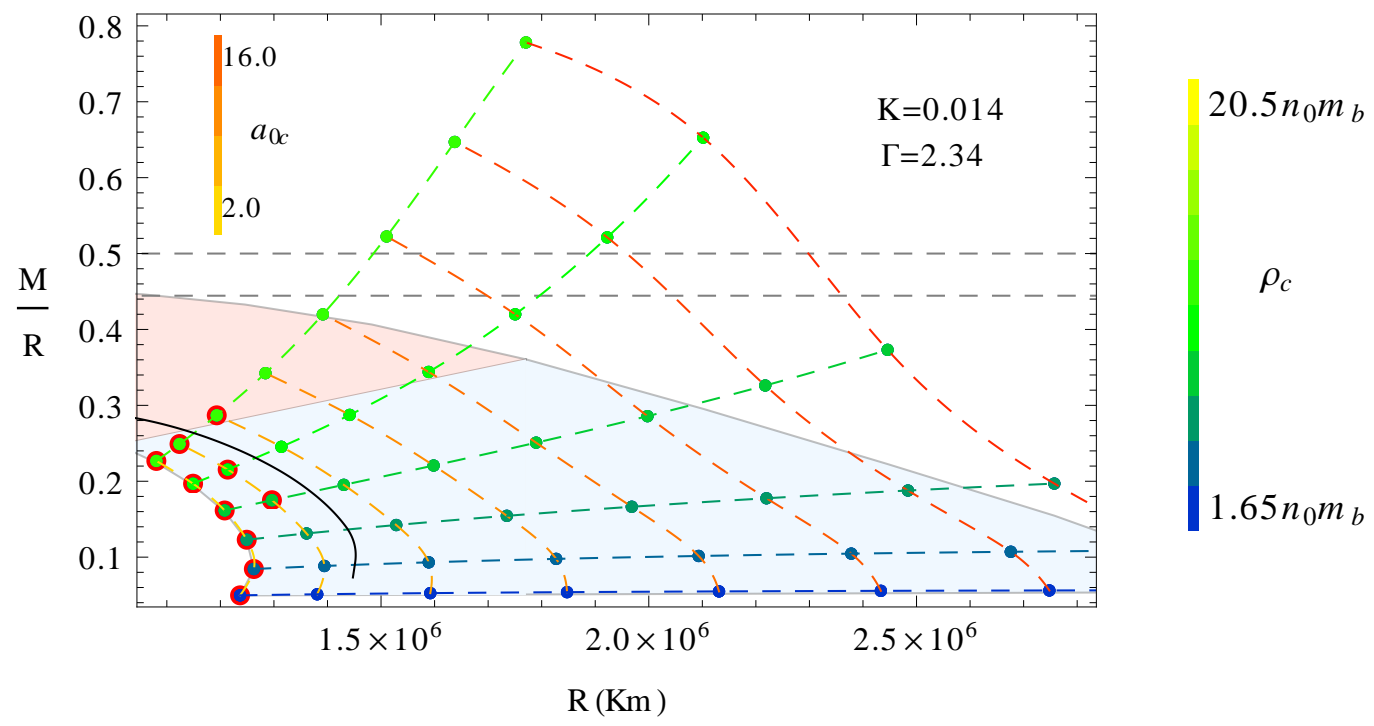

Figure 7: Compactness of NS. This quantity is computed with the Komar mass of the exterior solutions that match each NS configuration with the central value of $\rho$ and $A_{0}$ indicated by the color bars in the plot. Only stable configurations are shown. The black solid line shows the compactness of the same configurations in GR for polytropic stars modelled so that the M-R curve gives a maximum mass around $2 M_{\odot}$. For the vector Galileons we chose $K=0.014$ and $\Gamma=2.34$. The dashed horizontal lines show GR limits for the compactness of spherically symmetric and static solutions: $M / R=0.5$ is the compactness of Schwarzschild BH's, and $M / R=4 / 9$ is the compactness of incompressible stars. Notice that, thanks to the properties of our exterior solution, in some cases NS can be more compact than Schwarzschild black holes. For reference, the blue shaded region outlines instead the compactness of stable NS configurations if the exterior solution is set to be Schwarzschild geometry; the red shaded part extends this region to higher densities, where the solutions are unstable. These shaded regions show that NS with exterior configurations corresponding to Schwarzschild can not be more compact than black holes.

the colour bars in the plot: the initial condition $\rho_{c}$ is given by the point colour and the bar to the right of the plot, while $A_{0 c}$ is given by the dashed line on which the point resides and the colour bar inside the plot. The remaining initial conditions are $g_{t t}(0)=1, g_{t t}^{\prime}(0)=0$, and $A_{0}^{\prime}(0)=0$. The interior of the NS is modelled using a polytropic EoS with $K=0.014$ and $\Gamma=2.34$. This choice leads for $M-R$ curves with $M_{\max } \geq 2 M_{\odot}$ and real $\pi$. For the solutions circled in red, $\pi$ becomes complex inside the star. From each interior solution we obtain the radius $R$ of the respective star, which is given in the $x$-axis of the plot, and a set of initial conditions for the exterior solution. From the exterior solution we determine $M$. Knowing $R$ and $M$ we calculate the compactness of each star, and the results are shown in the $y$-axis of the plot.

Besides the compactness of NS in vector Galileons, Fig. (7) also displays the following reference lines: the dashed horizontal lines show GR limits for the compactness of spherically symmetric and static solutions: $M / R=0.5$ is the compactness of Schwarzschild BH's, and $M / R=4 / 9$ is the compactness of incompressible stars. EoS-dependent computations put lower limits on the compactness of NS, these are not shown in the plot. The black solid line shows the compactness of star configurations in the same range of densities shown in the plot but computed in GR and with polytropic parameters $K=0.0225$ and $\Gamma=2.34$, so that the M-R curve gives a maximum mass around $2 M_{\odot}$. The blue shaded region outlines the compactness of the same neutron stars solutions when we ignore the contributions of $A_{\mu}$ outside the star, i.e. we construct the global solution taking the same set of vector Galileon NS configurations corresponding to the points in the plot, but using a Schwarzschild configuration for the exterior metric. The red shading extends this to solutions with higher values of the central density, these solutions are unstable. As the plot shows, the solutions in the shaded region can never be more compact than a Schwarzschild black hole; this option is possible only for vector Galileons, thanks to the interactions between gravity and the vector field in the exterior of the star.

To conclude our discussion on neutron stars, we comment on possible future developments, in case the non- 
minimal vector-tensor couplings we consider is used to parameterize modifications of Maxwell electromagnetism in strong gravity regimes. While in this section we considered a situation in which matter in the NS interior does not directly couple to the vector (but only indirectly through gravity) we could directly couple $A_{\mu}$ with internal currents. It is known that for standard electromagnetism such couplings can modify the equilibrium configurations [33] since electric currents modify the equations of hydrostatic equilibrium. A complete treatment of this topic for vector Galileons is under investigation, and we include preliminary results in Appendix G. We find that, in certain cases, the inclusion of an interaction term $A_{\mu} J^{\mu}$ does not spoil the existence of neutron stars solutions in this model, and can improve the properties of the scalar field $\pi$, in such a way that configurations with complex $\pi$ (that we had to discard in our analysis above) can be turned into configurations with real scalar $\pi$. Other possible developments left for the future include the addition of rotation and magnetic fields (with $F_{i j} \neq 0$ ) to explore possible connections with magnestars. We make preliminary steps to find configurations with magnetic fields for vector Galileons in Appendix $F$, while rotating exterior configurations (for small values of the rotation parameter) are discussed in $[9,12]$. In the future, we plan to study whether $I$-Love- $Q$ relations $[34,35]$ - which relate the moment of inertia $I$, the tidal Love number, and the quadrupole moment $Q$ of the star - get modified in the context of vector Galileons.

\section{Discussion}

We studied black hole and neutron star configurations in a vector-tensor theory of gravity, a special case of vector Galileons, described by a simple, one parameter modification of the Einstein-Maxwell action, corresponding to the following non-minimal vector-tensor coupling

$$
\beta \sqrt{-g} G_{\mu \nu} A^{\mu} A^{\nu}
$$

with $G_{\mu \nu}$ the gravitational Einstein tensor and $\beta$ a coupling constant. Such coupling term can be used in the contexts of vector inflation, or a vector governing dark energy or dark matter. Alternatively, one may think of this vector as a way to parameterise deviations from the standard Maxwell's electromagnetism in regimes of strong gravity. The physics of black holes reveal surprisingly rich properties, that generalize the standard Reissner-Nordström solution of Einstein-Maxwell theory of gravity, but also black hole configurations of related scalar-tensor theories. There exist two disconnected branches of static, spherically symmetric, asymptotically flat solutions, whose features we studied in detail. Solutions have a mass $M$ and a vector charge $Q$, and are also characterized by an integration constant $P$ mainly controlling the profile of the longitudinal scalar polarisation of the vector $(P$ is not however associated to a conserved asymptotic charge). The existence and position of horizons depend in a non-trivial way by the parameters involved. In some cases analytic configurations are available, while for most values of the parameters involved we extract the behaviour of solutions through a numerical analysis. We find that in certain regions of parameter space black holes can be more compact than in General Relativity, providing a distinctive feature of black holes in this vector-tensor set-up. In an Appendix, moreover, we study in detail differences and similarities of our black hole configurations with solutions of scalar-tensor theories.

With respect to the neutron stars, our study shows that the vector profile plays an important role in determining the star configuration, both for controlling its internal hydrostatic equilibrium configuration, and for determining the external gravitational solution which is generally not described by a pure Schwarzschild geometry. The properties of neutron stars in this vector-tensor theory are quite rich; they can be larger and more massive than their GR counterparts and, for certain parameter choices, more compact. In some cases, they might be even more compact than Schwarzschild black holes, making this objects observationally interesting for the prospect of gravity wave detection. We also comment on possible generalizations to magnetically charged or rotating configurations, presenting in appendixes some preliminary calculations on this respect. This can be of interest especially when this vector-tensor non-minimal coupling is used to describe deviations from Maxwell theory, for describing exotic compact objects as magnetars.

Future interesting developments will include a study of rotating exterior solutions for neutron stars and black holes - with arbitrarily large rotation parameter - and an analysis of possible generalizations of I-Love-Q relations for neutron stars in vector Galileon theories. We hope to report soon on these topics.

\section{Acknowledgments}

JC is supported by STFC and CONACyt grants 263819 and 179208. GN is supported by CONACyT grants: 179208, 269652, Fronteras de la Ciencia 281, and by DAIP-Universidad de Guanajuato grant 1,046/2016. 


\section{Appendixes}

\section{A Black hole configurations for the branch $\pi=0$}

In this Appendix we discuss black hole configurations in the second branch of solutions satisfying the constraint (8) with $\pi=0$. This branch contains the Reissner-Nördstrom configuration in the limit $\beta=0$. However, for non-vanishing $\beta$, the profile for $A_{0}$ changes the geometry more drastically than in the other branch $\pi \neq 0$, leading to modifications of the GR geometry at large distances.

The algebraic constraint (8) vanishes identically for this branch, and the equations of motion (3a)-(3b) give two independent differential equations for $A_{0}$ and one of the metric functions $n$ and $m$. It is, however, more convenient to present the system as a set of three differential equations that are first order in derivatives of metric, namely

$$
\begin{aligned}
0= & r^{2} \kappa(r-2 m) A_{0}{ }^{2}-4\left(r-r \beta \kappa A_{0}^{2}-2 n\right) m^{\prime}, \\
0= & A_{0}{ }^{\prime}\left[r\left(n\left(-5+2 m^{\prime}\right)+r\left(2-m^{\prime}+n^{\prime}\right)\right)+m\left(8 n-r\left(3+2 n^{\prime}\right)\right)\right] \\
& +(r-2 n)\left(-4 \beta A_{0} m^{\prime}+r(r-2 m) A_{0}{ }^{\prime \prime}\right), \\
0= & n^{2}+2 r n\left(-2+2 \beta \kappa A_{0}{ }^{2}+8 r \beta \kappa A_{0} A_{0}{ }^{\prime}+r^{2} \kappa A_{0}{ }^{2}-4 n^{\prime}\right) \\
& -r^{2}\left(8 r \beta \kappa A_{0} A_{0}{ }^{\prime}+r^{2} \kappa A_{0}{ }^{2}-4 n^{\prime}+4 \beta \kappa A_{0}{ }^{2} n^{\prime}\right)+2 m\left[8 r \beta \kappa A_{0}(r-2 n) A_{0}{ }^{\prime}\right. \\
& \left.+(r-2 n)\left(2+r^{2} \kappa A_{0}{ }^{2}-4 n^{\prime}\right)+2 \beta \kappa A_{0}{ }^{2}\left(r-4 n+2 r n^{\prime}\right)\right] .
\end{aligned}
$$

The first (third) equation is algebraic in $n(m)$, and the result can be plugged back in the remaining two equations to find the two independent, but not first order in derivatives of the metric functions, equations for $m(n)$ and $A_{0}$.

We can not determine an analytical solution for this branch. We can proceed with studying solutions using perturbative expansions, as done for the first branch $\pi \neq 0$ in the main text.

\section{Asymptotic expansion for large values of the radial coordinate}

For $r \gg 1$ we apply the same perturbative approximations introduced in (16a)-(16b), and we make an expansion in a small $1 / r$ regime for $n(r), A_{0}(r)$, and $m(r)$. We find

$$
\begin{aligned}
1-\frac{2 n(r)}{r}= & -\epsilon \frac{2[M+\kappa \beta P(M P+2 Q)]}{r\left(1-P^{2} \beta \kappa\right)}+\frac{\epsilon^{2} \kappa}{2 r^{2}\left(1-P^{2} \beta \kappa\right)^{3}}\left\{Q^{2}(1-4 \beta)\right. \\
& -\kappa P^{2} \beta\left[Q^{2}+4\left(2 M^{2} P^{2}+2 M P Q-Q^{2}\right) \beta\right] \\
& \left.-8 \kappa^{2} P^{4} \beta^{3}(M P+Q)(M P+2 Q)\right\}+\mathcal{O}\left(\epsilon^{3}\right), \\
1-\frac{2 m(r)}{r}= & 1-\frac{2 M \epsilon}{r}+\frac{\epsilon^{2} Q^{2} \kappa}{2 r^{2}\left(1-\kappa \beta P^{2}\right)}+\mathcal{O}\left(\epsilon^{3}\right), \\
A_{0}(r)= & P+\frac{Q \epsilon}{r}-\frac{P Q(2 M P+Q) \beta \epsilon^{2} \kappa}{2 r^{2}\left(1-\kappa \beta P^{2}\right)}+\mathcal{O}\left(\epsilon^{3}\right) .
\end{aligned}
$$

In contrast to the case $\pi \neq 0$, the asymptotic geometry in this branch is more sensitive to the vector charge, which affects the geometry contributing already at first order in the expansion parameter $\epsilon$. It would be interesting to study in detail whether asymptotic scalar charges can characterise black hole configurations in this branch. Notice however that we could define a stronger weak-field limit, imposing that the parameter $P$, which enters at $r^{0}$ order in the expansion for $A_{0}$, is small: $P \rightarrow \epsilon P$. In this case, the corrections to the geometry associated with the vector contributions (parameters $P$ and $Q$ ) are pushed to second order in an $\epsilon$ expansion, and the geometry would be characterized by a Komar mass controlled by the parameter $M$.

\section{Small $\beta$ expansion}

This branch is smoothly connected to the $\mathrm{RN}$ solution in the limit $\beta \rightarrow 0$. The simplest way to see this is to consider the following ansatz:

$$
\begin{gathered}
1-\frac{2 n(r)}{r}=1-\frac{2 M}{r}+\beta f_{1}(r)+\beta^{2} f_{2}(r)+\ldots \\
1-\frac{2 m(r)}{r}=1-\frac{2 M}{r}+\beta g_{1}(r)+\beta^{2} g_{2}(r)+\ldots
\end{gathered}
$$




$$
A_{0}(r)=\sqrt{\beta} a_{1}(r)+\beta^{3 / 2} a_{2}(r)+\ldots,
$$

and solve for the functions $f_{1}, f_{2}, g_{1}, g_{2}, a_{1}, a_{2}, \ldots$ At leading order in $\beta$ the RN solution is generated:

$$
\begin{aligned}
g_{1}=f_{1} & =\frac{Q^{2}}{4 M_{p}^{2} r^{2}}, \\
a_{1} & =P-\frac{Q}{r} .
\end{aligned}
$$

This leading order solution perturbatively reconstruct the first term in the RN geometry. At next-to-leading order we find corrections only to the time component of the metric:

$$
\begin{aligned}
& g_{2}=a_{2}=0, \\
& f_{2}=-\frac{2 M P^{2}}{M_{p}^{2} r}+\frac{2 P Q}{M_{p}^{2} r}-\frac{Q^{2}}{M_{p}^{2} r^{2}} .
\end{aligned}
$$

It is possible to solve to higher orders in $\beta$, where corrections to $A_{0}$ and $g_{r r}$ are present too, however the analytic expressions are not particularly interesting. We verified that solving to the next orders in $\beta$ does not lead to new integration constants: any integration constants that arise at each higher order, can be reabsorbed in the definitions of $M, P$ and $Q$.

\section{B Exterior solutions in the branch $\pi \neq 0$}

In this appendix we numerically study exterior solutions for neutron star configurations, for different values of the parameter $\beta$ in the branch $\pi \neq 0$. Although we have in mind neutron stars, the same analysis remains valid outside the horizon of black hole configurations. Hence we study numerical solutions to eqs. $(11 \mathrm{a}, 11 \mathrm{~b})$ for $A_{0}(r)$ and $n(r)$ for representative values of $\beta$, and use eqs. $(9,10)$ to determine $\pi(r)$ and $m(r)$. We use c.g.s. units to ease the comparison to the literature on NS. To find numerical solutions, we set initial conditions at a typical radius of neutron stars, $R_{*}=12 \mathrm{Km}$, and take values for $n\left(R_{*}\right), n^{\prime}\left(R_{*}\right), A_{0}\left(R_{*}\right)$ and $A_{0}^{\prime}\left(R_{*}\right)$ within a range motivated by the interior numerical solutions that we investigate in the main text. From now on, all quantities with a subscript '*' denote quantities evaluated at $R_{*}$, for example $A_{0 *}=A_{0}\left(R_{*}\right)$. In order to work with dimensionless quantities, we introduce a normalisation for $A_{0}: a_{0}=A_{0} /\left(10^{23} \mathrm{~cm}^{1 / 2} \mathrm{gr}^{1 / 2} / \mathrm{s}\right)$, with $a_{0}$ dimensionless. This choice is made to match the order of magnitude of the quantity $1 / \sqrt{\kappa}=6.94 \times 10^{23} \mathrm{~cm}^{1 / 2} \mathrm{gr}^{1 / 2} / \mathrm{s}$ which turns out to have the same dimensions. Analogously, we also include a normalization for the scalar, $\pi_{N}=10^{23} \mathrm{~cm}^{1 / 2} \mathrm{gr}^{1 / 2} / \mathrm{s}$, and plot the dimensionless quantity $\pi / \pi_{N}$.

We plot our results for a representative choice of parameters in the Figure 8, and comment on their physical consequences below.

- Fig. 8a represents the metric components $g_{r r}^{-1}$ and $g_{t t}$ as function of the radial coordinates, for different choices of $\beta$ (the colour code for each line is explained at the bottom right panel of Fig. 8). When $\beta \neq 1 / 4$, these quantities are not equal. Asympototically, $g_{r r} \rightarrow 1$ in all cases, while in general $g_{t t}$ does not tend to unity at large $r$. Nevertheless, we can always redefine the time coordinate in such a way that $g_{t t}=1$ for large $r$, so to have an asymptotic configuration corresponding to Minkowski space written in spherical coordinates.

- Fig. 8b represents the radial evolution of mass of the object in units of Solar masses, for different choices of $\beta$. The asymptotic values of these quantity correspond to the Komar mass. While for $\beta=1 / 4$ the function $m(r)$ stops evolving outside the star, for different $\beta$ 's the vector field continues to source the Einstein tensor and consequently the gravitational mass is not fixed at $R_{*}$. As a result, the mass observed asymptotically in the models with $\beta<1 / 4$ is always larger than in GR. The models with large positive $\beta$ have to be discarded since $m$ takes negative asymptotic values. Indeed, we have checked that for large values of $\beta$ the solutions develop essential singularities at large values of $r$ outside the star surface, hence they are physically not interesting.

- Fig. $8 \mathrm{c}$ represents the radial profile of the time-like component of the vector field, $a_{0}=A_{0} /\left(10^{23} \mathrm{~cm}^{1 / 2} \mathrm{gr}^{1 / 2} / \mathrm{s}\right)$. For any $\beta \neq 1 / 4, A_{0}$ decays faster than for $\beta=1 / 4$ at large $r$; that is, it decays faster than $1 / r$. We do not find any hint of singular behavior in the numerical solutions for $A_{0}$. 

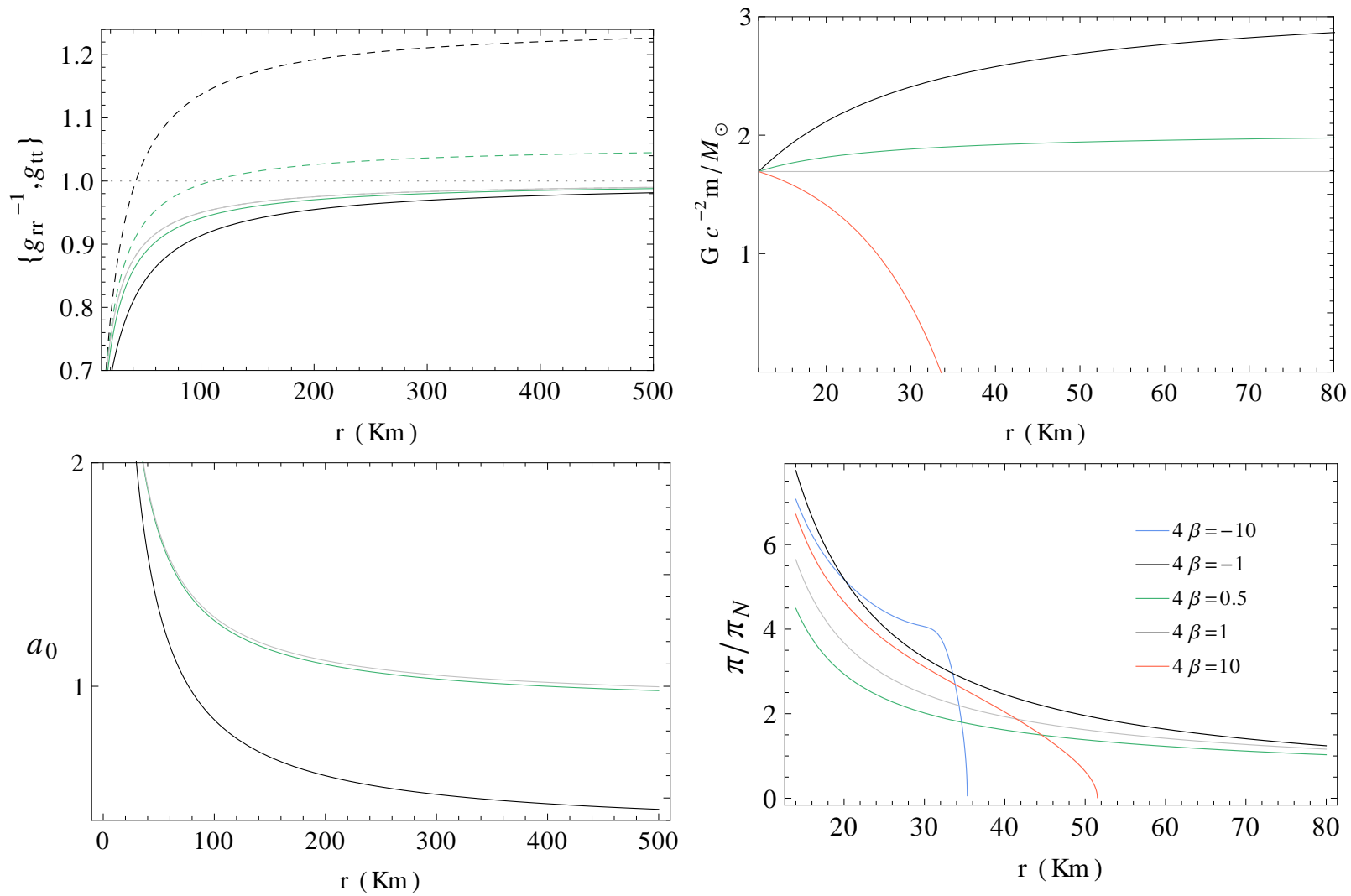

Figure 8: Clockwise from top-left, we represent various quantities as functions of radius $r$ : a) $g_{t t}$ (dashed lines) and $g_{r r}^{-1}$ (continuus line) components of the exterior metric; b) $m(r)$ measured in solar masses; c) $\left.a_{0}(r)=A_{0}(r) /\left(10^{23} \mathrm{~cm}^{1 / 2} \mathrm{gr}^{1 / 2} / \mathrm{s}\right) ; \mathrm{d}\right)$ the dimensionless quantity $\left|\pi(r) / \pi_{N}\right|$. The colour code in each plot is indicated in the bottom right panel. The horizontal line in the top left panel a) is a reference to make it easier to verify visually that $g^{r r} \rightarrow 1$ asymptotically. The initial conditions are $n_{*}=2.5 \mathrm{Km}, n_{*}^{\prime}=0, a_{0 *}=4, a_{0 *}^{\prime}=-0.27 \mathrm{Km}^{-1}$. See the main text for an analysis of the plots.

- Fig. 8d represents the radial profile for the absolute value of the scalar field $|\pi|$. While for small values of $\beta$ such radial profile is smooth, it develops singularities when $\beta$ is sufficiently large (of order 10 in absolute value), since such function becomes negative at finite values of $r$. This should be connected with the singularities in the geometry that we find for large values of $\beta$.

The results obtained indicate that $|\beta|$ cannot be arbitrarily large, otherwise the scalar field becomes complex.

\section{Difference between vector Galileon and scalar Horndenski theories}

In this appendix we discuss how black hole configurations in the vector-tensor (VT) system (2) we are considering differ from scalar-tensor (ST) theories as Horndeski. A comparison between these theories is not straightforward given the distinct nature of the fields involved. For example, in general $\nabla_{\mu} A_{\nu} \neq \nabla_{\nu} A_{\mu}$ unless $A_{\mu}=\partial_{\mu} \phi$ for some scalar field $\phi$. Furthermore, the number of equations of motion is in general different in the vector and scalar cases. The main message of this appendix will be that, when a comparison is possible, the vector Galileon model allows for physically interesting generalisations of the black hole solutions found in the Horndenski scalar case, also clarifying some of the properties of the latter.

\section{First difference: the set of equations}

Black hole configurations have been extensively studied in scalar-tensor theories. A particularly interesting subclass of scalar-tensor theories is known as the Fab Four [36], and has the property that it is the only subset of Horndeski's gravity where the cosmological constant can be self-tuned, meaning that Minkowski and cosmological solutions are 
allowed for any value of the bare cosmological constant. Within the Fab Four, there are minimal subsets that allow for self-tuning solutions while keeping some simplicity in their analytical treatment. An example is the action

$$
S=\int d^{4} x \sqrt{-g}\left[\tilde{\kappa}(R-2 \Lambda)-\frac{1}{2}\left(\tilde{\alpha} g^{\mu \nu}-\eta G^{\mu \nu}\right) \nabla_{\mu} \phi \nabla_{\nu} \phi\right]+S_{m},
$$

where $\tilde{\kappa}, \tilde{\alpha}$ and $\eta$ are coupling constants and $\phi$ a real scalar field. in the language of Fab Four, this corresponds to a combination of the George and John Lagrangians that has been studied in cosmological and static contexts (see for example [23,37]). When focussing on static spacetimes, the choice $\tilde{\alpha}=0$ and a scalar field profile with linear time dependence

$$
\phi(t, r)=P t+\psi(r)
$$

leads to a black hole solution with a Schwarzschild metric.

Eq. (42) is our starting point for a concrete comparison to the vector-tensor model: if we write $A_{\mu}=\partial_{\mu} \phi$, then $\partial_{r} \phi(t, r)=\partial_{r} \psi(r)$ plays the role of $\pi(r)$, and $\partial_{t} \phi(t, r)=P$ plays the role of a constant time like component $A_{0}$ of the vector field.

At first sight, one might think that under these identifications the vector system (2) is equivalent to (41) with $\tilde{\alpha}=0$ (remember that $F_{\mu \nu}$ vanishes for a constant $\left.A_{0}=P\right)$. However, this is not the case because the vector equation of motion derived from (2) is more restrictive than the scalar equation derived from (41): $\nabla_{\mu}\left(G^{\mu \nu} \partial_{\nu} \phi\right)=0$, where we set $\tilde{\alpha}=0$. While in the ST case only the vanishing of the radial component of $G^{\mu \nu} \partial_{\nu} \phi$ is required (by the $(t, r)$ component of the metric equations), in the VT case the time component of $G^{\mu \nu} \partial_{\nu} \phi$ is required to vanish as well, by the vector equations of motion. Under the spherically symmetric ansatz (5a), and with $A_{0}=P$, this last condition is $P m^{\prime}(r)=0$. Assuming $P \neq 0$, the only option left is $m(r)=$ constant, which leads to the stealthy Schwarzschild solution when in vacuum (see Section 3.1). Hence in vacuum, and with the simplest, constant profile for the vector $A_{0}$ component, ST and VT have the stealth Schwarzschild solution in common. The difference among the two systems arise in presence of matter: while in the ST case solutions with ansatz (42) can be found, in the VT with $A_{0}=P \neq 0$ the constraint $m^{\prime}=0$ forbids the existence of regular solutions.

Therefore, in the specific case $A_{0}=P$ the vector model is more restricted than the scalar model. On the other hand, the advantage of the VT model is that, as shown in the previous sections, we can turn on a non-trivial, radial dependent profile for $A_{0}$ and still find physically interesting vacuum solutions, including Schwarzschild, as well as physical solutions in presence of matter (as discussed in our analysis of neutron star configurations). These solutions do not have an equivalent in the scalar case: a static vector field with non-trivial profiles for the time and radial components is not obtainable in a ST theory (see also [12] for a related discussion).

\section{Second difference: black hole hairs}

Another physically relevant difference with respect to scalar-tensor theories lies in the "hair" of the spacetime solutions. In ST, there is a no-hair theorem for the shift-symmetric sub-sector of Horndeski gravity [38], a subsector that includes action (41). Under the assumptions that the spacetime is static, spherically symmetric and asymptotically flat, and the scalar field is a function only of $r$, regular $\mathrm{BH}$ solutions do not support non-trivial profiles of the scalar field (see $[3,23]$ for a concise review of the theorem and possible ways to circumvent it). Relaxing these assumptions BH's with scalar hair have been found [39], although this hair is dubbed "secondary" since it is not independent of the other charges (mass and electric charge for static BH's). Solutions with primary hair have been found in bi-scalar extensions of Horndeski gravity [40].

It is worth to clarify whether these no-hair theorems have any consequence for the vector model studied in this paper. When $A_{\mu}=\partial_{\mu} \phi$ the answer is yes, and the only BH that we can obtain is Schwarzschild. This is consistent with ST theories of the form (41) where, as discussed earlier, the only solution for $\tilde{\alpha}=0$ with a scalar field linear in $t$ is the Schwarzschild metric. As soon as we allow for a non-trivial profile of $A_{0}$, we break the relation $A_{\mu}=\partial_{\mu} \phi$ and we are able to find asymptotically flat BH solutions with primary hair, without introducing a time dependence in the vector field. This corresponds to the vector charge, denoted with $Q$ in the main text, analogously to Reissner-Nordström black holes. Recall that on the branch of solutions with a non-trivial profile for a scalar field $\pi \neq 0$, we have an independent integration constant (denoted with $P$ in the main text) which plays an important role in controlling the scalar profile. However, this integration constant is not associated with any conserved charge, and does not obey a Gauss law: hence it can not be considered a scalar hair. 


\section{Another class of vector Galileon black hole solutions}

For the sake of further comparison with the scalar tensor case, we can investigate what happens removing the term $F_{\mu \nu} F^{\mu \nu}$ in (2). This corresponds to a system that we do not consider in the rest of the paper, but which has the same Lagrangian structure as (41) with $\tilde{\alpha}=0$. In vacuum, for $A_{0}=P$ we have the stealth Schwarzschild solution. A more general solution with non-trivial $A_{0}$ exists, given by

$$
\begin{aligned}
n(r) & =m(r)=2 M, \\
A_{0}(r)^{2} & =P^{2}+\frac{2 P Q}{r}, \\
\pi(r)^{2} & =\frac{2 P(M P+Q) r}{(r-2 M)^{2}} .
\end{aligned}
$$

Asymptotically, the vector field profiles are

$$
\begin{aligned}
A_{0}(r) & =P+\frac{Q}{r}-\frac{Q^{2}}{2 P r^{2}}+\mathcal{O}\left(r^{-3}\right), \\
\pi(r) & =\sqrt{2 P(M P+Q)}\left(\frac{1}{r^{1 / 2}}+\frac{2 M}{r^{3 / 2}}+\mathcal{O}\left(r^{-5 / 2}\right)\right) .
\end{aligned}
$$

The origin of this solution is again in the vector equation of motion, which restricts the geometry to be Schwarzschild; and in the absence of the restriction $A_{\mu}=\partial_{\mu} \phi$ for some scalar field $\phi$.

\section{Charged black hole solutions for $\beta \approx 1 / 4$}

In this Appendix we provide more details on the arguments sketched in Section 3.2. We examine a region of interest in the available parameter space nearby the value $\beta=1 / 4$ in the branch $\pi \neq 0$. To search for solutions in this region, we express our fields as $n(r)=n_{0}+(\beta-1 / 4) n_{1}(r)+\ldots$, and similarly for $A_{0}, m$; then we solve the equations (11a)-(11b) order by order in $\beta-1 / 4$. We find

$$
\begin{aligned}
1-\frac{2 n(r)}{r} & =1-\frac{2 M}{r}+(\beta-1 / 4) \frac{8 Q^{2} \kappa}{r^{2}\left(\kappa P^{2}-4\right)}+\mathcal{O}\left[(\beta-1 / 4)^{2}\right], \\
1-\frac{2 m(r)}{r} & =1-\frac{2 M}{r}+(\beta-1 / 4)\left(1-\frac{M}{r}\right) \frac{16 Q^{2} \kappa}{r^{2}\left(\kappa P^{2}-4\right)}+\mathcal{O}\left[(\beta-1 / 4)^{2}\right], \\
A_{0}(r) & =P+\frac{Q}{r}+(\beta-1 / 4) \frac{4 P Q^{2} \kappa}{r^{2}\left(\kappa P^{2}-4\right)}+\mathcal{O}\left[(\beta-1 / 4)^{2}\right],
\end{aligned}
$$

$\pi$ can be determined from (10), resulting in the same expression as (12c), plus $\mathcal{O}(\beta-1 / 4)$-corrections.

In order to calculate reliably the position of the horizons $r_{h}$, located at the points where $g^{r r}=0$, we need to investigate whether these configurations can be trusted along the entire range of radial direction. To do so, we can estimate the size of higher order corrections $\mathcal{O}\left[(\beta-1 / 4)^{n}\right], n \geq 2$, to the previous formulae. The corrections of order $\mathcal{O}\left[(\beta-1 / 4)^{2}\right]$ are not difficult to calculate, and either by numerical analysis, or analytically but using some approximations ( $P$ and $M$ small). We find that for some values of the parameters $M, P$ and $Q$ the terms quadratic in $(\beta-1 / 4)$ become larger than terms linear on this quantity: there exists a critical value $r_{c}$ of the radial coordinate below which our expansion becomes unreliable. If $r_{h} \leq r_{c}$, our estimate of the position of horizons is unreliable. A full expression for $r_{c}$ in (45b) is complicated and not particularly illuminating. On the other hand, we can get intuition of its structure by considering the simplified case $P=0$ and $M / Q<<1$. Then we find that our series expansion is well defined up to second order in $(\beta-1 / 4)$, if

$$
3|\beta-1 / 4| A_{0}(r)^{2} \kappa \ll 1 .
$$

We then find an event horizon at the position

$$
r_{h} \approx 2 M+(\beta-1 / 4) \frac{4 \kappa Q^{2}}{4 M-\kappa M P^{2}}+\mathcal{O}\left[(\beta-1 / 4)^{2}\right] .
$$

as long as our choice of charges and parameters involved ensures that inequality (46) is satisfied at $r=r_{h}$. Expression (47) shows that the position of the horizons get indeed shifted when $\beta \neq 1 / 4$, by an amount depending also on 
the charges involved. Although there is no guarantee that the third and higher order terms in the series expansion (45a)-(45c) are also consistent under the conditions discussed above, we numerically checked that these findings are correct.

\section{E Black hole numerical solutions: the behavior of the charges}

When we discussed the parameter space for black hole solutions we used the parameters $\mathcal{P}$ and $\mathcal{Q}$ defined for numerical convenience as

$$
\begin{aligned}
& \mathcal{P}=A_{0}\left(r_{i}\right)+r_{i} A_{0}^{\prime}\left(r_{i}\right), \\
& \mathcal{Q}=-\frac{A_{0}^{\prime}\left(r_{i}\right)}{r_{i}^{2}} .
\end{aligned}
$$

These parameters correspond to the integration constants $P$ and $Q$ only when they are evaluated at $r_{i} \rightarrow \infty$. Numerically, we used $r_{i}=10^{3} \mathrm{Km}$. Here we show in what region of the parameter space this $r_{i}$ is large enough for $\mathcal{P}$ and $\mathcal{Q}$ to accurately represent $P$ and $Q$. To do so in a way that is easy to visualise we proceed as follows:

1. For each solution in Fig. 1 (which is reproduced in the left panel of Fig. 9) we solve from $r_{i}=10^{3} \mathrm{Km}$ outwards, up to $r_{a}=10^{9} \mathrm{Km}$, where we can guarantee that the fields have reached their asymptotic values.

2. At $r=r_{a}$ we compute $P$ and $Q$ for each solution and we redo Fig. 1 parametrising each solution in terms of $P$ and $Q$. This is shown in the right panel of Fig. 9, the colouring in this plot has the same meaning as in the left panel. The solutions in red in the left panel do not have a corresponding point in the right panel since they do not have an asymptotic region where $P$ and $Q$ can be defined

3. By comparing the left and right panels of Fig. 9 we get a qualitative idea of how different are $(\mathcal{P}, \mathcal{Q})$ and $(P, Q)$.

For most of the parameter space where regular solutions exist (R4), the "charges" evaluated close to the source are a good approximation for the asymptotic values of $P$ and $Q$. Stronger asymptotic effects could be present in the sub-regions of R4 where the blue points in the right panel of Fig. 9 are shifted with respect to the left panel, a more detailed numerical and analytical study of the solutions in those regions would be required to explore this.

\section{F Solutions with magnetic fields}

In this Appendix we present solutions to the system of vector Galileons (2) in presence of a $\varphi$-component of $A_{\mu}$ in the branches $\pi=0$ and $\pi \neq 0$ (which we interpret as 'magnetic field' since it turns on the spatial components $F_{i j}$ ). In both cases, our procedure consists in turning on $A_{\varphi}$ and identifying the metric components that are necessary to solve the equations of motion at the lowest order in an asymptotic approximation. We show that (2) admits a richer set of solutions when magnetic configurations are investigated.

\section{Branch $\pi \neq 0$}

In this case the component of the metric that we need to turn on to compensate for $A_{\varphi}$ is $g_{r \varphi}$.

$$
\begin{aligned}
1-\frac{2 n(r)}{r} & =1-\epsilon \frac{2 M}{r}, \\
1-\frac{2 m(r)}{r} & =1-\epsilon \frac{2 M}{r}, \\
g_{r \varphi}(r, \theta) & =\epsilon^{3 / 2} \frac{2 \sqrt{2} B \sqrt{M P^{2}+Q P} \beta \epsilon^{3 / 2} \kappa \cos \theta}{\sqrt{r}\left(1+P^{2} \beta \kappa\right)}, \\
A_{0}(r) & =P+\epsilon \frac{Q}{r}, \\
\pi(r) & =\epsilon^{1 / 2} \frac{\sqrt{2} \sqrt{M P^{2}+Q P}}{\sqrt{r}},
\end{aligned}
$$



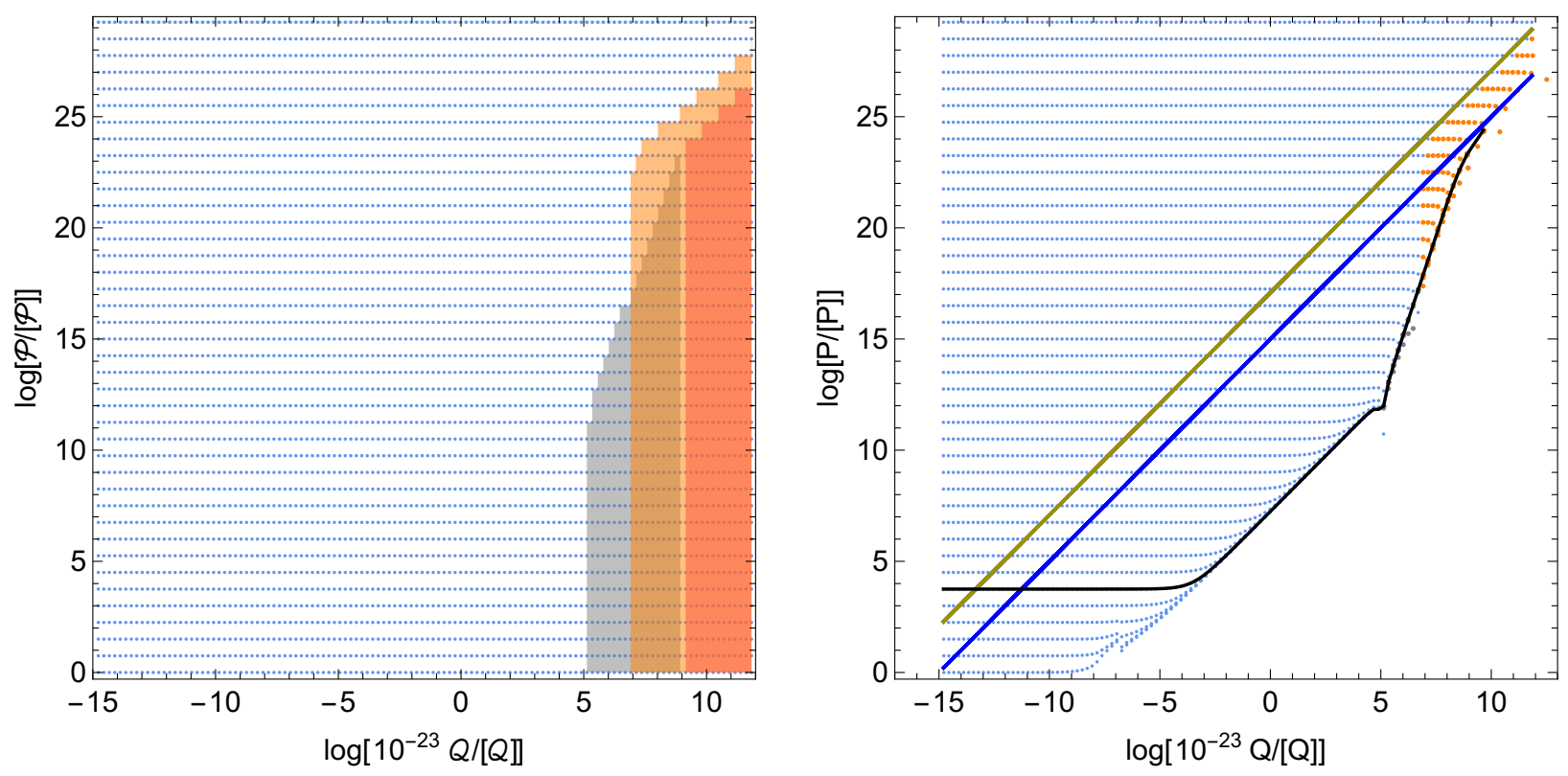

Figure 9: Black hole behaviour in terms of the vector field charges. The axes in the left panel are parametrised in terms of quantities evaluated close to the source, $\mathcal{P}$ and $\mathcal{Q}$, while in the right panel the axes represent the asymptotic quantities $Q$ and $P$. The blue solid line corresponds to $\mathcal{P}=\mathcal{Q} / r_{i}$, and the brown solid line to $M=P / Q$. The reasons to show these lines are: for solutions below the blue line we naively expect that $\mathcal{Q}$ is large enough to cause a difference between $\mathcal{P}$ and $P$, or, in other words, that an asymptotic expansion of the form $P+Q / r$ is not yet valid at $r_{i}$. As for the brown line, when $P$ and $Q$ have opposite signs - which is not the case in this plot - solutions below the line $|M|=|P| /|Q|$ are expected to have a complex $\pi$. This does not happen for this set of solutions, but interestingly enough, for large $P$ and $Q$ the same line signals the transition between regular solutions and solutions without an event horizon. In these plots we fixed $\mathcal{M}=4.5 M_{\odot}$. The asymptotic mass $M$ is not fixed, when the vector charges are large the difference between $M$ and $\mathcal{M}$ is at most $5 \%$, i.e. $1-M /\left(4.5 M_{\odot}\right) \approx 0.05$.

$$
A_{\varphi}(r, \theta)=\epsilon B \cos \theta
$$

The metric is still static, but the cross term $G_{r \varphi}$ induces a deformation of the spatial line element that breaks spherical symmetry, as can be confirmed by computing the invariants $R_{\mu \nu} R^{\mu \nu}$ and $R_{\mu \nu \alpha \beta} R^{\mu \nu \alpha \beta}$ and noticing that they depend on $r$ and $\theta$. Indeed, the Kretschmann scalar is the same as for the Schwarzschild geometry to leading order in $\epsilon$, the angular dependence enters at next-to-leading order and gives a contribution of the form $w(\theta) / r^{7}$, where $w(\theta)$ is a function only of $\theta$ that diverges near $\theta=0$ and $\theta=\pi$, signalling that near the poles our approximation is only valid for $r \rightarrow \infty$.

\section{Branch $\pi=0$}

Here we need to turn on the metric components $g_{t \varphi}$ and $g_{r, \theta}$, and the effect of the magnetic field on the other fields is only seen at quadratic and higher orders in $\epsilon$. For simplicity, we set $P=0$, but we checked that an approximated solution can be found with small $P$ as well. For large $P$, the time component of the metric acquires a complicated angular dependence.

$$
\begin{aligned}
1-\frac{2 n(r)}{r} & =1-\epsilon \frac{2 M}{r}+\epsilon^{2} \kappa \frac{B^{2}+Q^{2}(1-4 \beta)}{2 r^{2}} \\
1-\frac{2 m(r)}{r} & =1-\epsilon \frac{2 M}{r}+\epsilon^{2} \kappa \frac{Q^{2}+B^{2}(1+2 \beta)-2 B^{2} \beta h(\theta)}{2 r^{2}} \\
h(\theta) & =\csc ^{2} \theta+\cos \theta \log \cot (\theta / 2) \\
g_{r \theta}(r, \theta) & =\epsilon^{2} \kappa \beta B^{2} \frac{\left(11 \cos \theta-3 \cos (3 \theta)+4 \log \tan (\theta / 2) \sin ^{4} \theta\right)}{8 r \sin ^{3} \theta} \\
g_{t \phi}(r, \theta) & =\epsilon^{2} \frac{2 \kappa \beta B Q \cos \theta}{r}
\end{aligned}
$$




$$
\begin{aligned}
A_{0}(r) & =\epsilon \frac{Q}{r}, \\
A_{\varphi}(r, \theta) & =\epsilon B \cos \theta .
\end{aligned}
$$

Once again, the curvature invariants depend on $r$ and $\theta$ and near the poles the approximation is only valid if $r \rightarrow \infty$. This branch is connected to the magnetic RN solution in the limit $\beta \rightarrow 0$.

\section{G Currents inside the star: the basic formalism}

The system (2) can be generalised in order to explore the consequences of adding a current density term, so that the action now reads

$$
S=\int d^{4} x \sqrt{-g}\left[\frac{1}{2 \kappa}(R-2 \Lambda)-\frac{1}{4} F^{\mu \nu} F_{\mu \nu}+\beta G_{\mu \nu} A^{\mu} A^{\nu}+j^{\mu} A_{\mu}+\mathcal{L}_{\text {matter }}\right] .
$$

For simplicity, the current density is chosen to be proportional to the matter density,

$$
j^{\mu}=\sqrt{\kappa} \gamma \rho(r) U^{\mu}
$$

where $U^{\mu}$ is the 4 -velocity of the perfect fluid described by $T_{\mu \nu}$. In the rest frame of the perfect fluid - the same that we use to write the components of $T_{\mu \nu}$ - the only non-vanishing component of the 4 -velocity is $U^{t}=\sqrt{g^{t t}}$, where $g^{t t}$ comes from (5). $\kappa$ is introduced for convenience to make the arbitrary constant $\gamma$ dimensionless: $U^{\mu}$ is dimensionless, $[\rho]=\left[c^{2}\right] \cdot$ mass $/$ lenght ${ }^{3}=\left[\kappa^{-1}\right]\left[\ell^{-2}\right]$, and $j^{\mu}$ has to have dimensions of $\left[A^{\mu}\right]\left[\ell^{-2}\right]=\left[\kappa^{-1 / 2}\right]\left[\ell^{-2}\right]$, these dimensions are given by $\sqrt{\kappa} \rho$. The dimensions of $j^{\mu}$ can be written as (electric charge)/length ${ }^{3}$, therefore the quantity $c^{2} j^{0} / \rho(r)=c^{2} \sqrt{\kappa} \gamma U^{0}$ is a mass-to-charge ratio characteristic of the solutions under consideration. Since $U^{0}<<1$, the maximum mass-to-charge ratio is attained at the center of the star and given by $c^{2} \sqrt{\kappa} \gamma$.

Let us derive the equations of motion. The term $\sqrt{-g} j^{\mu}$ is constant under variation of the metric. Therefore, $j^{\mu} A_{\mu}$ only contributes to the vector equation of motion:

$$
0=D^{\mu} F_{\mu \nu}+2 \beta G_{\mu \nu} A^{\mu}+j_{\nu}
$$

Note that, whatever the form of $j^{\mu}$ - i.e., forget (53) for a moment, the angular components of this equation demand $j^{\theta}=j^{\varphi}=0$. In addition, in the branch $\pi \neq 0$ we need to satisfy (9) (the only assumptions behind this equation, are that $T_{\mu \nu}$ is a perfect fluid described in its rest frame and that the metric is diagonal). Once (9) is substituted in (54), the $r$-component of such equations demands $j^{r}=0$. Thus, $j^{0}$ is the only new function introduced in (52).

Restoring (53), and supplementing our system with the EoS (30b), we have a complete set of equations to determine $A_{0}(r), n(r)$ and $\chi(r)$. The only difference with respect to the system analysed in Sec. 4 is the contribution of $j^{0}$ to the vector equation.

Although not necessary -since all the information is encoded in the system of equations described above - it is illuminating to combine the Bianchi identity with the vector equation in such a way that the physical difference between the systems described by actions (2) and (52) is highlighted:

$$
0=j_{0} A_{0}^{\prime}-p^{\prime}-\frac{n-r n^{\prime}}{r^{2}-2 r n}(p+\rho) .
$$

Eq. (55) shows that the inclusion of a current density allows the vector field to affect directly the equation that determines the matter equilibrium configurations. This equation is known as the hydroelectrostatic equilibrium equation.

Further physical insight can be gained by rewriting the $t$-component of the vector equation of motion as

$$
0=j_{0}(r)-\frac{1}{r^{2}} \sqrt{-g^{t t} g^{r r}}\left[r^{2} \sqrt{-g_{t t} g_{r r}} F^{t r}\right]^{\prime}-\beta \frac{2 g^{t t} A_{0}}{r^{2}}\left[r\left(1-g^{r r}\right)\right]^{\prime},
$$

where $F^{t r}=-g^{t t} g^{r r} A_{0}^{\prime}$. The last expression can be formally integrated once to give

$$
0=\Theta(r)-r^{2} \sqrt{-g_{t t} g_{r r}} F^{t r}-\int \frac{2 \beta A_{0}}{\sqrt{-g_{t t} g^{r r}}}\left[r\left(1-g^{r r}\right)\right]^{\prime} d r,
$$

where an integration constant has been absorbed in $\Theta(r)=\int_{0}^{r} r^{2} \sqrt{-g_{t t} g_{r r}} j_{0} d r+\Theta_{0}$. Notice that since $j_{0} \neq 0$ only inside a source, $\Theta=\Theta_{0}$ for exterior solutions. The last term in (57) is in general non-vanishing, $A_{0}$ sources the electromagnetic tensor and consequently the gravitational fields in and outside the star. Far from the source, in asymptotically flat spacetime, (57) gives as solution $A_{0}=\Theta_{0} / r+$ const., thus $\theta_{0}$ can be identified with the asymptotic charge $Q$. The asymptotic corrections discussed around eq. (17c) originate from the last term in (57). 


\section{References}

[1] B. P. Abbott et al. [LIGO Scientific and Virgo Collaborations], Phys. Rev. Lett. 116 (2016) no.6, 061102 doi:10.1103/PhysRevLett.116.061102 [arXiv:1602.03837 [gr-qc]].

[2] T. Clifton, P. G. Ferreira, A. Padilla and C. Skordis, Phys. Rept. 513, 1 (2012) doi:10.1016/j.physrep.2012.01.001 [arXiv:1106.2476 [astro-ph.CO]].

[3] C. A. R. Herdeiro and E. Radu, Int. J. Mod. Phys. D 24 (2015) no.09, 1542014 doi:10.1142/S0218271815420146 [arXiv:1504.08209 [gr-qc]].

[4] E. Berti et al., Class. Quant. Grav. 32 (2015) 243001 doi:10.1088/0264-9381/32/24/243001 [arXiv:1501.07274 [gr-qc]].

[5] A. Golovnev, V. Mukhanov and V. Vanchurin, JCAP 0806 (2008) 009 doi:10.1088/1475-7516/2008/06/009 [arXiv:0802.2068 [astro-ph]]; M. a. Watanabe, S. Kanno and J. Soda, Phys. Rev. Lett. 102 (2009) 191302 doi:10.1103/PhysRevLett.102.191302 [arXiv:0902.2833 [hep-th]].

[6] B. Holdom, Phys. Lett. 166B (1986) 196. doi:10.1016/0370-2693(86)91377-8; N. Arkani-Hamed, D. P. Finkbeiner, T. R. Slatyer and N. Weiner, Phys. Rev. D 79 (2009) 015014 doi:10.1103/PhysRevD.79.015014 [arXiv:0810.0713 [hep-ph]]; L. Ackerman, M. R. Buckley, S. M. Carroll and M. Kamionkowski, Phys. Rev. D 79 (2009) 023519 doi:10.1103/PhysRevD.79.023519, 10.1142/97898142937920021 [arXiv:0810.5126 [hep-ph]].

[7] G. Tasinato, JHEP 1404, 067 (2014) doi:10.1007/JHEP04(2014)067 [arXiv:1402.6450 [hep-th]]. B. M. Gripaios, JHEP 0410, 069 (2004) doi:10.1088/1126-6708/2004/10/069 [hep-th/0408127]. L. Heisenberg, JCAP 1405, 015 (2014) doi:10.1088/1475-7516/2014/05/015 [arXiv:1402.7026 [hep-th]].

[8] G. Tasinato, Class. Quant. Grav. 31 (2014) 225004 doi:10.1088/0264-9381/31/22/225004 [arXiv:1404.4883 [hep-th]]; M. Hull, K. Koyama and G. Tasinato, JHEP 1503 (2015) 154 doi:10.1007/JHEP03(2015)154 [arXiv:1408.6871 [hep-th]]; M. Hull, K. Koyama and G. Tasinato, Phys. Rev. D 93 (2016) no.6, 064012 doi:10.1103/PhysRevD.93.064012 [arXiv:1510.07029 [hep-th]]; E. Allys, P. Peter and Y. Rodriguez, JCAP 1602 (2016) no.02, 004 doi:10.1088/1475-7516/2016/02/004 [arXiv:1511.03101 [hep-th]]; J. Chagoya and G. Tasinato, JHEP 1602 (2016) 063 doi:10.1007/JHEP02(2016)063 [arXiv:1511.07805 [hep-th]]; A. De Felice, L. Heisenberg, R. Kase, S. Tsujikawa, Y. 1. Zhang and G. B. Zhao, Phys. Rev. D 93 (2016) no.10, 104016 doi:10.1103/PhysRevD.93.104016 [arXiv:1602.00371 [gr-qc]]; J. Beltran Jimenez and L. Heisenberg, Phys. Lett. B 757 (2016) 405 doi:10.1016/j.physletb.2016.04.017 [arXiv:1602.03410 [hep-th]]; A. De Felice, L. Heisenberg, R. Kase, S. Mukohyama, S. Tsujikawa and Y. l. Zhang, JCAP 1606 (2016) no.06, 048 doi:10.1088/1475-7516/2016/06/048 [arXiv:1603.05806 [gr-qc]].

[9] J. Chagoya, G. Niz and G. Tasinato, Class. Quant. Grav. 33, no. 17, 175007 (2016) doi:10.1088/0264-9381/33/17/175007 [arXiv:1602.08697 [hep-th]].

[10] G. Tasinato, K. Koyama and G. Niz, Class. Quant. Grav. 30, 184002 (2013) doi:10.1088/0264-9381/30/18/184002 [arXiv:1304.0601 [hep-th]].

[11] J. D. Bekenstein, Phys. Rev. D 5 (1972) 1239. doi:10.1103/PhysRevD.5.1239; J. D. Bekenstein, Phys. Rev. D 5 (1972) 2403. doi:10.1103/PhysRevD.5.2403

[12] M. Minamitsuji, Phys. Rev. D 94, no. 8, 084039 (2016) doi:10.1103/PhysRevD.94.084039 [arXiv:1607.06278 [gr-qc]].

[13] E. Babichev, C. Charmousis and M. Hassaine, arXiv:1703.07676 [gr-qc].

[14] D. G. Boulware and S. Deser, "String Generated Gravity Models," Phys. Rev. Lett. 55 (1985) 2656. doi:10.1103/PhysRevLett.55.2656

[15] A. Salam and J. A. Strathdee, "A Class of Solutions for the Strong Gravity Equations," Phys. Rev. D 16 (1977) 2668. doi:10.1103/PhysRevD.16.2668

[16] A. Chamblin, R. Emparan, C. V. Johnson and R. C. Myers, Phys. Rev. D 60, 064018 (1999) doi:10.1103/PhysRevD.60.064018 [hep-th/9902170].

[17] K. Koyama, G. Niz and G. Tasinato, Phys. Rev. Lett. 107 (2011) 131101 doi:10.1103/PhysRevLett.107.131101 [arXiv:1103.4708 [hep-th]]; K. Koyama, G. Niz and G. Tasinato, Phys. Rev. D 84 (2011) 064033 doi:10.1103/PhysRevD.84.064033 [arXiv:1104.2143 [hep-th]].

[18] S. M. Carroll, San Francisco, USA: Addison-Wesley (2004) 513 p 
[19] H. van Dam and M. J. G. Veltman, Nucl. Phys. B 22, 397 (1970). doi:10.1016/0550-3213(70)90416-5 V. I. Zakharov, JETP Lett. 12 (1970) 312 [Pisma Zh. Eksp. Teor. Fiz. 12 (1970) 447].

[20] C. Charmousis, G. Niz, A. Padilla and P. M. Saffin, JHEP 0908, 070 (2009) doi:10.1088/1126-6708/2009/08/070 [arXiv:0905.2579 [hep-th]].

[21] M. C. Miller and J. M. Miller, Phys. Rept. 548 (2014) 1 doi:10.1016/j.physrep.2014.09.003 [arXiv:1408.4145 [astroph.HE]].

[22] T. Damour and G. Esposito-Farese, Phys. Rev. Lett. 70 (1993) 2220. doi:10.1103/PhysRevLett.70.2220

[23] A. Maselli, H. O. Silva, M. Minamitsuji and E. Berti, Phys. Rev. D 93 (2016) no.12, 124056 doi:10.1103/PhysRevD.93.124056 [arXiv:1603.04876 [gr-qc]].

[24] A. Cisterna, T. Delsate and M. Rinaldi, Phys. Rev. D 92 (2015) no.4, 044050 doi:10.1103/PhysRevD.92.044050 [arXiv:1504.05189 [gr-qc]]. A. Cisterna, T. Delsate, L. Ducobu and M. Rinaldi, Phys. Rev. D 93 (2016) no.8, 084046 doi:10.1103/PhysRevD.93.084046 [arXiv:1602.06939 [gr-qc]].

[25] P. Pani, E. Berti, V. Cardoso and J. Read, Phys. Rev. D 84, 104035 (2011) doi:10.1103/PhysRevD.84.104035 [arXiv:1109.0928 [gr-qc]].

[26] E. Babichev, C. Charmousis and A. Lehbel, "Black holes and stars in Horndeski theory," Class. Quant. Grav. 33 (2016) no.15, 154002 doi:10.1088/0264-9381/33/15/154002 [arXiv:1604.06402 [gr-qc]].

[27] T. Damour and G. Esposito-Farese, Phys. Rev. D 54 (1996) 1474 doi:10.1103/PhysRevD.54.1474 [gr-qc/9602056].

[28] P. Demorest, T. Pennucci, S. Ransom, M. Roberts and J. Hessels, Nature 467 (2010) 1081 doi:10.1038/nature09466 [arXiv:1010.5788 [astro-ph.HE]].

[29] J. Antoniadis et al., Science 340 (2013) 6131 doi:10.1126/science.1233232 [arXiv:1304.6875 [astro-ph.HE]].

[30] P. Haensel, A. Y. Potekhin and D. G. Yakovlev, Astrophys. Space Sci. Libr. 326 (2007). doi:10.1007/978-0-387-47301-7

[31] C. E. Rhoades, Jr. and R. Ruffini, Phys. Rev. Lett. 32 (1974) 324. doi:10.1103/PhysRevLett.32.324

[32] C. M. Will, Cambridge University Pres, 1993.

[33] J. D. Bekenstein, Phys. Rev. D 4 (1971) 2185. doi:10.1103/PhysRevD.4.2185

[34] K. Yagi and N. Yunes, "I-Love-Q Relations in Neutron Stars and their Applications to Astrophysics, Gravitational Waves and Fundamental Physics," Phys. Rev. D 88 (2013) no.2, 023009 doi:10.1103/PhysRevD.88.023009 [arXiv:1303.1528 $[\mathrm{gr}-\mathrm{qc}]]$.

[35] K. Yagi and N. Yunes, Science 341 (2013) 365 doi:10.1126/science.1236462 [arXiv:1302.4499 [gr-qc]].

[36] C. Charmousis, E. J. Copeland, A. Padilla and P. M. Saffin, Phys. Rev. Lett. 108, 051101 (2012) doi:10.1103/PhysRevLett.108.051101 [arXiv:1106.2000 [hep-th]].

[37] J. P. Bruneton, M. Rinaldi, A. Kanfon, A. Hees, S. Schlogel and A. Fuzfa, Adv. Astron. 2012, 430694 (2012) doi:10.1155/2012/430694 [arXiv:1203.4446 [gr-qc]]. M. Rinaldi, Phys. Rev. D 86, 084048 (2012) doi:10.1103/PhysRevD.86.084048 [arXiv:1208.0103 [gr-qc]]. G. Koutsoumbas, K. Ntrekis, E. Papantonopoulos and M. Tsoukalas, Phys. Rev. D 95, no. 4, 044009 (2017) doi:10.1103/PhysRevD.95.044009 [arXiv:1512.05934 [gr-qc]]. H. O. Silva, A. Maselli, M. Minamitsuji and E. Berti, "Compact objects in Horndeski gravity," Int. J. Mod. Phys. D 25 (2016) no.09, 1641006 doi:10.1142/S0218271816410066 [arXiv:1602.05997 [gr-qc]].

[38] L. Hui and A. Nicolis, Phys. Rev. Lett. 110, 241104 (2013) doi:10.1103/PhysRevLett.110.241104 [arXiv:1202.1296 [hep-th]].

[39] E. Babichev and C. Charmousis, JHEP 1408 (2014) 106 doi:10.1007/JHEP08(2014)106 [arXiv:1312.3204 [gr-qc]].

[40] C. Charmousis, T. Kolyvaris, E. Papantonopoulos and M. Tsoukalas, JHEP 1407, 085 (2014) doi:10.1007/JHEP07(2014)085 [arXiv:1404.1024 [gr-qc]]. 